doi: $10.13108 / 2016-8-4-3$

UDC 517.5

\title{
REPRESENTATION OF ANALYTIC FUNCTIONS
}

\section{A.I. ABDULNAGIMOV, A.S. KRIVOSHEYEV}

\begin{abstract}
In this paper we consider exponential series with complex exponents, whose real and imaginary parts are integer. We prove that each function analytical in the vicinity of the closure of a bounded convex domain in the complex plain can be expanded into the above mentioned series and this series converges absolutely inside this domain and uniformly on compact subsets. The result is based on constructing a regular subset with a prescribed angular density of the sequence of all complex numbers, whose real and imaginary parts are integer.
\end{abstract}

Keywords: analytic function, exponential series, regular set, density of sequence.

Mathematics Subject Classification 30D10

\section{INTRODUCTION}

Let $\Lambda_{\mathbb{Z}}=\left\{\lambda_{k}\right\}_{k=1}^{\infty}$ be a sequence of all complex numbers with integer coordinates $\lambda_{k}=m+i l$, $m, l \in \mathbb{Z}$, taken in the order of ascending absolute values. We consider the series

$$
\sum_{m, l \in \mathbb{Z}} d_{m, l} e^{(m+i l) z}
$$

We assume that this series converges at each point of some open subset $E \subset \mathbb{C}$. It is easy to see that the sequence $\Lambda_{\mathbb{Z}}=\left\{\lambda_{k}\right\}_{k=1}^{\infty}$ satisfies the relations

$$
\lim _{k \rightarrow \infty} \frac{\ln k}{\left|\lambda_{k}\right|}=\lim _{k \rightarrow \infty} \frac{\ln k}{\sqrt{k}}=0 .
$$

Then according to the results of work [1] (Theorems 3.1 and 4.1), series (1.1) converges in a convex domain $D \subset \mathbb{C}$ containing the convex hull of the set $E$. The domain $D$ is determined by means of Cauchy-Hadamard formula for the series of exponentials (see [1, Thm. 4.1]):

$$
\begin{aligned}
& D=\{z \in \mathbb{C}: \operatorname{Re}(z \xi)<h(\xi),|\xi|=1\}, \\
& h(\xi)=\inf \varliminf_{j \rightarrow \infty} \frac{\ln \left(1 /\left|d_{m(k(j)), l(k(j))}\right|\right)}{\lambda_{k(j)}}, \quad \lambda_{k}=m(k)+i l(k),
\end{aligned}
$$

where the infimum is taken over all the subsequences $\left\{\lambda_{k(j)}\right\}_{j=1}^{\infty}$ of the sequence $\Lambda_{\mathbb{Z}}$ such that $\lambda_{k(j)} /\left|\lambda_{k(j)}\right|$ converges to the point $\xi$ as $j \rightarrow \infty$. At that, series (1.1) converges absolutely and uniformly on compact subsets of the domain $D$ (see [1, Thm. 3.1]). Therefore, its sum $g(z)$ is an analytic function in the domain $D$.

This, if series (1.1) converges on an open subset $E \subset \mathbb{C}$, it converges on a convex domain $D$ containing $E$ to a function analytic in $D$.

In the present work we solve an inverse in some sense problem on representing each function analytic in the vicinity of the closure of an arbitrary fixed bounded convex domain $D \subset \mathbb{C}$ by series (1.1) converging on $D$.

A.I. Abdulnagimov, A.S. Krivosheyev, Representation of analytic functions.

(c) Abdulnagimov A.I., Krivosheyev A.S. 2016.

Submitted April 17, 2016. 
Thanks to a classical result by A.F. Leontiev (see [2, Ch. IV, Sect. 6, Thm. 4.6.4]) on representation of functions analytic in the vicinity of the closure of a bounded convex domain $D \subset \mathbb{C}$, we succeed to reduce the mentioned problem to the problem on constructing a regular set with a prescribed angular density (see [3, Ch. II, Sect. 1]) being a part of the sequence $\Lambda_{\mathbb{Z}}$.

In the second section (Thms. 2.1 and 2.2), the latter problem is solved completely. On the base of this problem, in the third section we prove (Theorem 3.2) that each function analytic in the vicinity of the closure of an arbitrary fixed bounded convex domain $D \subset \mathbb{C}$ is expanded into series (1.1) converging absolutely and uniformly on compact subsets of the domain $D$.

\section{Constructing of Regular SeT}

Let $\Lambda=\left\{\lambda_{k}\right\}_{k=1}^{\infty}$ be a sequence of complex numbers taken in the order of ascending absolute values such that $\left|\lambda_{k}\right| \rightarrow \infty$. We denote by $n(r, \Lambda)$ the amount of points $\lambda_{k}$ lying in the ball $B(0, r)$ of radius $r>0$ centered at the origin. The upper and lower densities of $\Lambda$ are respectively the quantities

$$
\underline{n}(\Lambda)=\lim _{r \rightarrow \infty} \frac{n(r, \Lambda)}{r}, \quad \bar{n}(\Lambda)=\varlimsup_{r \rightarrow \infty} \frac{n(r, \Lambda)}{r} .
$$

The sequence $\Lambda$ is said to have a density $n(\Lambda)$ (measurable) if $\underline{n}(\Lambda)=\bar{n}(\Lambda)=n(\Lambda)<+\infty$. We have:

$$
\begin{aligned}
& \varlimsup_{k \rightarrow \infty} \frac{k}{\left|\lambda_{k}\right|} \leqslant \varlimsup_{k \rightarrow \infty} \frac{n\left(\left|\lambda_{k}\right|+1, \Lambda\right)}{\left|\lambda_{k}\right|}=\varlimsup_{k \rightarrow \infty} \frac{n\left(\left|\lambda_{k}\right|+1, \Lambda\right)}{\left|\lambda_{k}\right|+1} \leqslant \bar{n}(\Lambda), \\
& \varliminf_{k \rightarrow \infty} \frac{k}{\left|\lambda_{k}\right|} \geqslant \varliminf_{k \rightarrow \infty} \frac{n\left(\left|\lambda_{k}\right|-1, \Lambda\right)}{\left|\lambda_{k}\right|}=\varliminf_{k \rightarrow \infty} \frac{n\left(\left|\lambda_{k}\right|-1, \Lambda\right)}{\left|\lambda_{k}\right|-1} \geqslant \underline{n}(\Lambda) .
\end{aligned}
$$

Thus, if sequence $\Lambda$ is measurable, then

$$
n(\Lambda)=\lim _{k \rightarrow \infty} \frac{k}{\left|\lambda_{k}\right|} .
$$

Let $\varphi_{1}, \varphi_{2} \in[-2 \pi, 2 \pi), \varphi_{2}-\varphi_{1} \in(0,2 \pi]$. We shall call such values $\varphi_{1}, \varphi_{2}$ admissible. We let

$$
\Gamma\left(\varphi_{1}, \varphi_{2}\right)\left(\Gamma\left(\varphi_{1}, \varphi_{2}\right]\right)=\left\{\lambda=t e^{i \varphi}: \varphi \in\left(\varphi_{1}, \varphi_{2}\right)\left(\left(\varphi_{1}, \varphi_{2}\right]\right), t>0\right\} .
$$

By the symbol $\Lambda\left(\varphi_{1}, \varphi_{2}\right)\left(\Lambda\left(\varphi_{1}, \varphi_{2}\right]\right)$ we denote the sequence consisting of all pairs $\left\{\lambda_{k}, n_{k}\right\}$ such that $\lambda_{k} \in \Gamma\left(\varphi_{1}, \varphi_{2}\right)\left(\Gamma\left(\varphi_{1}, \varphi_{2}\right]\right)$.

Lemma 2.1. Let $\varphi_{1}, \varphi_{2}$ be admissible and $\gamma>0$. There exists $R_{0}>0$ such that as $R>R_{0}$, each interval $(R, R+\gamma)$ contains the absolute value $\left|\lambda_{k}\right|$ of some point $\lambda_{k} \in \Lambda_{\mathbb{Z}}\left(\varphi_{1}, \varphi_{2}\right)$.

Proof. Let $p, m$ be integer number such that the ray $\Gamma$ starting at the origin and passing the point with the coordinates $(m, p)$ lies (except the beginning) in the angle $\Gamma\left(\varphi_{1}, \varphi_{2}\right)$. Such $p$, $m$ can be chosen by taken an appropriate approximation of the number $\tan \varphi$ by the fractions $p / m\left(\varphi \in\left(\varphi_{1}, \varphi_{2}\right) \backslash\{\pi k\}_{k \in \mathbb{Z}}\right.$ is chosen arbitrarily).

The ray $\Gamma$ consists of the diagonals of the rectangles, whose vertices are the points in the sequences $\Lambda_{\mathbb{Z}}$, while the lengths of the sides are equal to $|p|$ and $|m|$. It contains the points $\lambda_{k(s)}$, $s=1,2, \ldots$, of the sequence $\Lambda_{\mathbb{Z}}\left(\varphi_{1}, \varphi_{2}\right)$ separated by the distance $h$. The latter coincides with the lengths of the diagonals of the mentioned rectangles. The points $\lambda_{k(s)}$ have the coordinates $(s m, s p), s=1,2, \ldots$ Let $\varsigma_{s}$ be the straight line perpendicular to the ray $\Gamma$ and passing through the points $\lambda_{k(s)}, s \geqslant 1$. It also consists of the diagonals of the rectangles, whose vertices are the points in the sequence $\Lambda_{\mathbb{Z}}$ and the lengths of their sides are equal to $|p|$ and $|m|$. For each $s \geqslant 1$, the straight line $\varsigma_{s}$ contains the points $\lambda_{k(s, j)}, j \in \mathbb{Z}$, of the sequence $\Lambda_{\mathbb{Z}}$. Their are of the form $\lambda_{k(s, j)}=\lambda_{k(s)}+j h e^{i\left(\varphi_{0}+\pi / 2\right)}, j \in \mathbb{Z}$, where $\varphi_{0}$ is determined by the identity $\tan \varphi_{0}=p / m$.

Since the ray $\Gamma \backslash\{0\}$ lies in the angle $\Gamma\left(\varphi_{1}, \varphi_{2}\right)$, for some number $\beta>0$ each point $\lambda_{k(s, j)}$ obeying the condition $0 \leqslant j \leqslant \beta_{s}$ and $s \geqslant 1$ belongs to the sequence $\Lambda_{\mathbb{Z}}\left(\varphi_{1}, \varphi_{2}\right)$. Let $s \geqslant 1$ and $j_{s}$ be the maximal number $j \geqslant 0$ satisfying the inequality $j \leqslant \beta_{s}$. 
We consider the set $\left|\lambda_{k(s, j)}\right|, j=0,1, \ldots, j_{s}$. The quantity $\left|\lambda_{k(s, j)}\right|$ is the hypotenuse of the right triangle with the vertices $0, \lambda_{k(s)}$ and $\lambda_{k(s, j)}$. The length $\left|\lambda_{k(s)}-\lambda_{k(s, j)}\right|$ of one of its cathetus is an increasing function in the parameter $j \geqslant 0$; it is equal to $j h$. This is why we get the inequalities

We have

$$
\left|\lambda_{k(s, 0)}\right|<\left|\lambda_{k(s, 1)}\right|<\cdots<\left|\lambda_{k\left(s, j_{s}\right)}\right|
$$

$$
\left|\lambda_{k(s, 0)}\right|=\left|\lambda_{k(s)}\right|=\sqrt{(s m)^{2}+(s p)^{2}}=s \sqrt{m^{2}+s^{2}}=s h
$$

and as $j \geqslant 0$,

$$
\begin{aligned}
\left|\lambda_{k(s, j+1)}\right|-\left|\lambda_{k(s, j)}\right| & =\sqrt{\left|\lambda_{k(s)}\right|^{2}+((j+1) h)^{2}}-\sqrt{\left|\lambda_{k(s)}\right|^{2}+(j h)^{2}} \\
& =\sqrt{(s h)^{2}+((j+1) h)^{2}}-\sqrt{(s h)^{2}+(j h)^{2}} \\
& =h\left(\sqrt{s^{2}+(j+1)^{2}}-\sqrt{s^{2}+j^{2}}\right)=h \sqrt{s^{2}+j^{2}}\left(\sqrt{1+\frac{2 j+1}{s^{2}+j^{2}}}-1\right) .
\end{aligned}
$$

We have the estimate $\ln \sqrt{1+x} \leqslant x / 2$. Therefore,

$$
\sqrt{1+x} \leqslant e^{x / 2}=\sum_{n=0}^{\infty} \frac{x^{n}}{2^{n} n !} \leqslant 1+\frac{x}{2}+x^{2} \sum_{n=0}^{\infty} x^{n}=1+\frac{x}{2}+\frac{x^{2}}{1-x} \leqslant 1+x
$$

for all $x \in[0,1 / 4]$. By the above relations we obtain

$$
\left|\lambda_{k(s, j+1)}\right|-\left|\lambda_{k(s, j)}\right| \leqslant h \sqrt{s^{2}+j^{2}} \frac{2 j+1}{s^{2}+j^{2}}=h \frac{2 j+1}{\sqrt{s^{2}+j^{2}}}=h \frac{2 j+1}{s \sqrt{1+(j / s)^{2}}} \leqslant h \frac{2 j+1}{s} .
$$

We choose an index $s_{0}$ such that $h / s_{0}<\gamma / 2$. Then

$$
\left|\lambda_{k(s, j+1)}\right|-\left|\lambda_{k(s, j)}\right|<\gamma, \quad j \leqslant \frac{s \gamma}{4 h}, \quad s \geqslant s_{0} .
$$

Let $\alpha=\min \{\beta, s \gamma / 4 h\}$ and $\widetilde{j}_{s}$ be the maximal index $j \geqslant 0$ satisfying the inequality $j \leqslant \beta s$. Then $\widetilde{j}_{s} \leqslant j_{s}$. This is why all the points $\lambda_{k(s, j)}, j=\overline{0, \widetilde{j}_{s}}, s \geqslant 1$, belong to the sequence $\Lambda_{\mathbb{Z}}\left(\varphi_{1}, \varphi_{2}\right)$. Moreover, the inequalities

$$
\left|\lambda_{k(s, j+1)}\right|-\left|\lambda_{k(s, j)}\right|<\gamma, \quad j=\overline{0, \widetilde{j}_{s}}, \quad s \geqslant s_{0} .
$$

Let us estimate from below the quantity $\left|\lambda_{k\left(s, \widetilde{j}_{s}\right)}\right|$. By the choice of the index $\widetilde{j}_{s}$ we have

$$
\left|\lambda_{k\left(s, \widetilde{j}_{s}\right)}\right|=\sqrt{\left|\lambda_{k(s)}\right|^{2}+\left(\widetilde{j}_{s} h\right)^{2}}=\sqrt{(s h)^{2}+((\beta s-1) h)^{2}}=s h \sqrt{1+\left(\frac{\beta s-1}{s}\right)^{2}} .
$$

Since

$$
\lim _{s \rightarrow \infty} \frac{s}{s+1} \sqrt{1+\left(\frac{\beta s-1}{s}\right)^{2}}=\sqrt{1+\beta^{2}}>1
$$

there exists an index $s_{1} \geqslant s_{0}$ such that

$$
\left|\lambda_{k\left(s, \widetilde{j}_{s}\right)}\right|>s h++h, \quad s \geqslant s_{1}
$$

Thus, for each $s \geqslant s_{1}$ we an increasing set of numbers $\left|\lambda_{k(s, j)}\right|, j=\overline{0, \widetilde{j}_{s}}$ such that $\lambda_{k(s, j)} \in$ $\Lambda_{\mathbb{Z}}\left(\varphi_{1}, \varphi_{2}\right)$ for all $j=\overline{0, \widetilde{j}_{s}}$. The first of these numbers coincides with $s h$, while the other is strictly greater than $s h+h$. In view of $(2.1)$ it follows that each interval $(R, R+\gamma)$ intersecting the semi-interval $[s h, s h+h)$ contains at least one of the numbers in this set. Letting now $R_{0}=s_{1} h$, we complete the proof of the lemma. 
Let $\Lambda^{1}=\left\{\lambda_{k}^{1}\right\}_{k=1}^{\infty}$ and $\Lambda^{2}=\left\{\lambda_{j}^{2}\right\}_{j=1}^{\infty}$. We shall say that $\Lambda^{1}$ is a subsequence of $\Lambda^{2}$ and we shall write $\Lambda^{1} \subset \Lambda^{2}$ if there exists a set of the indices $j(k), k \geqslant 1$, such that $\lambda_{k}^{1}=\lambda_{j(k)}^{2}, k \geqslant 1$. If $\lambda_{k}^{1} \neq \lambda_{j}^{2}, k, j \geqslant 1$, then by the union $\Lambda^{1} \cup \Lambda^{2}$ of the sequences $\Lambda^{1}$ and $\Lambda^{2}$ we shall mean the sequence consisting of all elements $\lambda_{k}^{1}, \lambda_{j}^{2}, k, j \geqslant 1$.

The proof of the next statement is based on the method exposed in the proof of Lemma 5 in work [4], see also [5, Lm. 2].

Lemma 2.2. Let $\varphi_{1}, \varphi_{2}$ be admissible, $\delta>0$ and $\Lambda^{0}=\left\{\lambda_{k}^{0}\right\}_{k=1}^{\infty}$ have a density $\tau_{0} \geqslant 0$ (probably, $\Lambda^{0}$ is empty). Assume that $\tau>\tau_{0}$. Then there exists a sequence $\Lambda^{1}=\left\{\lambda_{j}^{1}\right\}_{j=1}^{\infty} \subseteq$ $\Lambda_{\mathbb{Z}}\left(\varphi_{1}, \varphi_{2}\right)$ having density $\tau-\tau_{0}$ such that

$$
|| \lambda_{j}^{1}|-| \lambda_{k}^{0}|| \geqslant \frac{1}{2 \tau}-\frac{\delta}{2}, \quad k, j \geqslant 1, \quad\left|\lambda_{j+1}^{1}\right|-\left|\lambda_{j}^{1}\right| \geqslant \frac{1}{\tau}-\delta, \quad j \geqslant 1 .
$$

If $\Lambda^{0}$ is empty, the first inequality in (2.2) is omitted.

Proof. Let $\alpha=1 / \tau$ and $\gamma=\min \{\delta, \alpha\}$. By Lemma 2.1, there exists a natural number $p_{0}$ such that as $R \geqslant p_{0} \alpha$, each interval $(R, R+\gamma)$ contains the absolute value $\left|\xi_{m}\right|$ of some point $\xi_{m} \in \Lambda_{\mathbb{Z}}\left(\varphi_{1}, \varphi_{2}\right)$. We seek the sequence $\Lambda^{1}$ as the union $\Lambda^{1}=\bigcup_{p \geqslant p_{0}} \Lambda_{p}^{1}$. We construct the sets $\Lambda_{p}^{1}, p \geqslant p_{0}$, by the induction. First we denote the total amount of the points in the set $\Lambda_{s}^{1}$, $s=\overline{p_{0}, p}$, by the symbol $N_{p}$.

Let $p=p_{0}$. If the semi-interval $[p \alpha,(p+1) \alpha)$ contains at least one of the elements of the sequence $\left\{\left|\lambda_{k}^{0}\right|\right\}_{k=1}^{\infty}$, we let $\Lambda_{p}^{1}=\emptyset$. Otherwise as $\Lambda_{p}^{1}$ we choose the set consisting of one point $\xi_{m} \in \Lambda_{\mathbb{Z}}\left(\varphi_{1}, \varphi_{2}\right)$, whose absolute value $\left|\xi_{m}\right|$ belongs to the interval

$$
\left(\frac{2 p+1}{2} \alpha-\frac{\gamma}{2}, \frac{2 p+1}{2} \alpha+\frac{\gamma}{2}\right) \text {. }
$$

At least one such point $\xi_{m}$ exists by the definition of the point $p_{0}$; if there are several such points, we arbitrarily choose one of them.

Suppose now $p>p_{0}$. We assume that we have constructed the sets $\Lambda_{s}^{1}$ for all $s=\overline{p_{0}, p-1}$. Let us define $\Lambda_{p}^{1}$. If the inequality

$$
N_{p-1}+n\left((p+1) \alpha, \Lambda^{0}\right)-n\left(p_{0} \alpha, \Lambda^{0}\right) \geqslant p+1-p_{0}
$$

holds true, we let $\Lambda_{p}^{1}=\emptyset$. If

$$
N_{p-1}+n\left((p+1) \alpha, \Lambda^{0}\right)-n\left(p_{0} \alpha, \Lambda^{0}\right)<p+1-p_{0}
$$

as $\Lambda_{p}^{1}$ we choose a set consisting of some point $\xi_{m} \in \Lambda_{\mathbb{Z}}\left(\varphi_{1}, \varphi_{2}\right)$ whose absolute value $\left|\xi_{m}\right|$ belongs to interval (2.3). As above, there exists at least one such point $\xi_{m}$.

Thus, we have constructed the sequence $\Lambda^{1}$. Let us show that it is the sought one. We let $\Lambda=\Lambda^{0} \cup \Lambda^{1}$ and we are going to prove that $\Lambda$ has density $\tau$.

Let us prove first that for all $l>p_{0}$ the inequalities

$$
l-p_{0} \leqslant n(l \alpha, \Lambda)-n\left(p_{0} \alpha, \Lambda\right) \leqslant \max \left\{l-p_{0}, N_{l-2}+n\left(l \alpha, \Lambda^{0}\right)-n\left(p_{0} \alpha, \Lambda^{0}\right)\right\}
$$

hold true, where for the convenience we let $N_{p_{0}-1}=0$.

If as $p=l-1$, we have (2.4), then by the construction $\Lambda_{l-1}^{1}=\emptyset$. This is why $N_{l-1}=N_{l-2}$. Therefore,

$$
n(l \alpha, \Lambda)-n\left(p_{0} \alpha, \Lambda\right)=N_{l-1}+n\left(l \alpha, \Lambda^{0}\right)-n\left(p_{0} \alpha, \Lambda^{0}\right)=N_{l-2}+n\left(l \alpha, \Lambda^{0}\right)-n\left(p_{0} \alpha, \Lambda^{0}\right),
$$

that is, the right inequality in (2.6) holds true. If (2.5) holds true, then by the construction $\Lambda_{l-1}^{1}$ consists of one point. This is why $N_{l-1}=N_{l-2}+1$. Therefore, by (2.5) we have

$$
\begin{aligned}
n(l \alpha, \Lambda)-n\left(p_{0} \alpha, \Lambda\right) & =N_{l-1}+n\left(l \alpha, \Lambda^{0}\right)-n\left(p_{0} \alpha, \Lambda^{0}\right) \\
& =N_{l-2}+1+n\left(l \alpha, \Lambda^{0}\right)-n\left(p_{0} \alpha, \Lambda^{0}\right)<l-p_{0}+1 \leqslant l-p_{0} .
\end{aligned}
$$


Thus, the right inequality in (2.6) holds true in this case as well.

Let us prove the left inequality. We employ the induction. If the semi-interval $\left[p_{0} \alpha,\left(p_{0}+1\right) \alpha\right)$ contains at least one element of the sequence $\left\{\left|\lambda_{k}^{0}\right|\right\}_{k=1}^{\infty}$, then

$$
n\left(\left(p_{0}+1\right) \alpha, \Lambda\right)-n\left(p_{0} \alpha, \Lambda\right) \geqslant n\left(\left(p_{0}+1\right) \alpha, \Lambda^{0}\right)-n\left(p_{0} \alpha, \Lambda^{0}\right) \geqslant 1=p_{0}+1-p_{0},
$$

that is, in this case the left inequality in (2.6) holds true. If the semi-interval $\left[p_{0} \alpha,\left(p_{0}+1\right) \alpha\right)$ contains no elements of the sequence $\left\{\left|\lambda_{k}^{0}\right|\right\}_{k=1}^{\infty}$, by the construction, $\Lambda_{p_{0}}^{1}$ consists of one point. This is why

$$
n\left(\left(p_{0}+1\right) \alpha, \Lambda\right)-n\left(p_{0} \alpha, \Lambda\right) \geqslant n\left(\left(p_{0}+1\right) \alpha, \Lambda^{1}\right)-n\left(p_{0} \alpha, \Lambda^{1}\right)=N_{p_{0}}=1=p_{0}+1-p_{0} .
$$

Thus, the left inequality in (2.6) is true under the assumption $l=p_{0}+1$. We assume that it is true for all $l=\overline{p_{0}+1, p}$ and let us prove it for $l=p+1$.

If (2.4) holds true for $p=l-1$, then

$$
\begin{aligned}
n(l \alpha, \Lambda)-n\left(p_{0} \alpha, \Lambda\right) & =n((p+1) \alpha, \Lambda)-n\left(p_{0} \alpha, \Lambda\right)=N_{p}+n\left((p+1) \alpha, \Lambda^{0}\right)-n\left(p_{0} \alpha, \Lambda^{0}\right) \\
& \geqslant N_{p-1}+n\left((p+1) \alpha, \Lambda^{0}\right)-n\left(p_{0} \alpha, \Lambda^{0}\right) \geqslant p+1-p_{0}=l-p_{0},
\end{aligned}
$$

that is, in this case the left inequality in (2.6) holds true. Assume that (2.5) is true for $p=l-1$. Then by the definition $\Lambda_{p}^{1}$ consists of one point, that is, $N_{p}=N_{p-1}+1$. By the induction assumption $n(p \alpha, \Lambda)-n\left(p_{0} \alpha, \Lambda\right) \geqslant p-p_{0}$. Therefore,

$$
\begin{aligned}
n(l \alpha, \Lambda)-n\left(p_{0} \alpha, \Lambda\right) & =n((p+1) \alpha, \Lambda)-n\left(p_{0} \alpha, \Lambda\right) \\
& =n(p \alpha, \Lambda)-n\left(p_{0} \alpha, \Lambda\right)++n((p+1) \alpha, \Lambda)-n(p \alpha, \Lambda) \\
& \geqslant p-p_{0}+n\left((p+1) \alpha, \Lambda^{1}\right)-n\left(p \alpha, \Lambda^{1}\right)=p-p_{0}+N_{p}-N_{p-1} \\
& =p-p_{0}+1=l-p_{0} .
\end{aligned}
$$

Thus, inequalities (2.6) are proved.

Employing the left inequality in (2.6), we obtain

$$
\begin{aligned}
\underline{n}(\Lambda) & =\varliminf_{r \rightarrow \infty} \frac{n(r, \Lambda)}{r} \geqslant \underline{\lim _{l \rightarrow \infty}} \frac{n(l \alpha, \Lambda)}{(l+1) \alpha}=\varliminf_{l \rightarrow \infty} \frac{n(l \alpha, \Lambda)}{l \alpha} \\
& =\varliminf_{l \rightarrow \infty} \frac{n(l \alpha, \Lambda)-n\left(p_{0} \alpha, \Lambda\right)+n\left(p_{0} \alpha, \Lambda\right)}{l \alpha}=\varliminf_{l \rightarrow \infty} \frac{n(l \alpha, \Lambda)-n\left(p_{0} \alpha, \Lambda\right)}{l \alpha} \\
& \geqslant \varliminf_{l \rightarrow \infty} \frac{l-p_{0}}{l \alpha}=\varliminf_{l \rightarrow \infty} \frac{l}{l \alpha}=\frac{1}{\alpha}=\tau .
\end{aligned}
$$

It implies that $\underline{n}(\Lambda) \geqslant \tau$. Let us prove the inequality $\bar{n}(\Lambda) \leqslant \tau$.

Let $r_{j} \rightarrow \infty$ be a sequence realizing the uppoer limit in the definition of the quantity $\bar{n}(\Lambda)$, and $l(j), j \geqslant 1$, be the minimal natural number such that $l(j) \alpha \geqslant r_{j}$. If $n(l \alpha, \Lambda)-n\left(p_{0} \alpha, \Lambda\right)>l-p_{0}$ for all $l>p_{0}$, by the right inequality in (2.6) and the definition of $N_{l}$ we have

$$
\begin{aligned}
n(l \alpha, \Lambda)-n\left(p_{0} \alpha, \Lambda\right) & \leqslant N_{l-2}+n\left(l \alpha, \Lambda^{0}\right)-n\left(p_{0} \alpha, \Lambda^{0}\right) \\
& =n((l-1) \alpha, \Lambda)-n\left(p_{0} \alpha, \Lambda\right)+n\left(l \alpha, \Lambda^{0}\right)-n\left((l-1) \alpha, \Lambda^{0}\right) \\
& \leqslant N_{l-3}+n\left((l-1) \alpha, \Lambda^{0}\right)-n\left(p_{0} \alpha, \Lambda^{0}\right)++n\left(l \alpha, \Lambda^{0}\right)-n\left((l-1) \alpha, \Lambda^{0}\right) \\
& =n((l-2) \alpha, \Lambda)-n\left(p_{0} \alpha, \Lambda\right)++n\left(l \alpha, \Lambda^{0}\right)-n\left((l-2) \alpha, \Lambda^{0}\right) \\
& \leqslant \cdots \\
& \leqslant N_{p_{0}-1}+n\left(\left(p_{0}+1\right) \alpha, \Lambda^{0}\right)-n\left(p_{0} \alpha, \Lambda^{0}\right)+n\left(l \alpha, \Lambda^{0}\right)-n\left(\left(p_{0}+1\right) \alpha, \Lambda^{0}\right) \\
& =n\left(l \alpha, \Lambda^{0}\right)-n\left(p_{0} \alpha, \Lambda^{0}\right) .
\end{aligned}
$$


Hence, taking into consideration that $\Lambda^{0}$ has the density $\tau_{0}<\tau$, we obtain:

$$
\begin{aligned}
\bar{n}(\Lambda) & =\lim _{j \rightarrow \infty} \frac{n\left(r_{j}, \Lambda\right)}{r_{j}} \leqslant \varlimsup_{j \rightarrow \infty} \frac{n(l(j) \alpha, \Lambda)}{(l(j)-1) \alpha}=\varlimsup_{j \rightarrow \infty} \frac{n(l(j) \alpha, \Lambda)-n\left(p_{0} \alpha, \Lambda\right)+n\left(p_{0} \alpha, \Lambda\right)}{l(j) \alpha} \\
& =\varlimsup_{j \rightarrow \infty} \frac{n(l(j) \alpha, \Lambda)-n\left(p_{0} \alpha, \Lambda\right)}{l(j) \alpha} \leqslant \varlimsup_{j \rightarrow \infty} \frac{n\left(l(j) \alpha, \Lambda^{0}\right)-n\left(p_{0} \alpha, \Lambda^{0}\right)}{l(j) \alpha} \\
& =\varlimsup_{j \rightarrow \infty} \frac{n\left(l(j) \alpha, \Lambda^{0}\right)}{l(j) \alpha} \leqslant \bar{n}\left(\Lambda^{0}\right)=\tau_{0}<\tau .
\end{aligned}
$$

This contradicts the inequality $\underline{n}(\Lambda) \geqslant \tau$. Therefore, $n(l \alpha, \Lambda)-n\left(p_{0} \alpha, \Lambda\right) \leqslant l-p_{0}$ at least for one index $l>p_{0}$. Thus, there exists $j_{0}$ such that for each $j \geqslant j_{0}$ there exists a maximal natural number $m(j)$ satisfying the conditions: $m(j) \leqslant l(j)$ and $n(m(j) \alpha, \Lambda)-n\left(p_{0} \alpha, \Lambda\right) \leqslant m(j)-p_{0}$. Choosing a subsequence, we can assume that $m(j) / l(j)$ converges to some number $\gamma \in[0,1]$. Employing the right inequality in (2.6), as above, we obtain the estimate

$$
\begin{aligned}
n(l(j) \alpha, \Lambda)-n\left(p_{0} \alpha, \Lambda\right) & \leqslant n((l(j)-1) \alpha, \Lambda)-n\left(p_{0} \alpha, \Lambda\right)+n\left(l(j) \alpha, \Lambda^{0}\right)-n\left((l(j)-1) \alpha, \Lambda^{0}\right) \\
& \leqslant \cdots \leqslant n(m(j) \alpha, \Lambda)-n\left(p_{0} \alpha, \Lambda\right)+n\left(l(j) \alpha, \Lambda^{0}\right)-n\left(m(j) \alpha, \Lambda^{0}\right) .
\end{aligned}
$$

By the choice of index $m(j)$ we get

$$
\begin{aligned}
\bar{n}(\Lambda) & \leqslant \varlimsup_{j \rightarrow \infty} \frac{n(l(j) \alpha, \Lambda)}{(l(j)-1) \alpha}=\varlimsup_{j \rightarrow \infty} \frac{n(l(j) \alpha, \Lambda)}{l(j) \alpha}=\varlimsup_{j \rightarrow \infty} \frac{n(l(j) \alpha, \Lambda)-n\left(p_{0} \alpha, \Lambda\right)}{l(j) \alpha} \\
& \leqslant \varlimsup_{j \rightarrow \infty} \frac{n(m(j) \alpha, \Lambda)-n\left(p_{0} \alpha, \Lambda\right)}{l(j) \alpha}+\varlimsup_{j \rightarrow \infty} \frac{n\left(l(j) \alpha, \Lambda^{0}\right)-n\left(m(j) \alpha, \Lambda^{0}\right)}{l(j) \alpha} \\
& \leqslant \varlimsup_{j \rightarrow \infty} \frac{m(j)-p_{0}}{l(j) \alpha}+\varlimsup_{j \rightarrow \infty} \frac{n\left(l(j) \alpha, \Lambda^{0}\right)-n\left(m(j) \alpha, \Lambda^{0}\right)}{l(j) \alpha} \\
& =\frac{\gamma}{\alpha}+\varlimsup_{j \rightarrow \infty} \frac{n\left(l(j) \alpha, \Lambda^{0}\right)-n\left(m(j) \alpha, \Lambda^{0}\right)}{l(j) \alpha} .
\end{aligned}
$$

If $\gamma=0$, then

$$
\bar{n}(\Lambda) \leqslant \varlimsup_{j \rightarrow \infty} \frac{n\left(l(j) \alpha, \Lambda^{0}\right)-n\left(m(j) \alpha, \Lambda^{0}\right)}{l(j) \alpha} \leqslant \varlimsup_{j \rightarrow \infty} \frac{n\left(l(j) \alpha, \Lambda^{0}\right)}{l(j) \alpha} \leqslant \bar{n}\left(\Lambda^{0}\right)=\tau_{0}<\tau .
$$

This contradicts the inequality $\underline{n}(\Lambda) \geqslant \tau$. Therefore, $\gamma>0$. We choose $\delta^{\prime}>0$ such that $\gamma-\delta^{\prime}>0$. We let $\delta=1-\gamma+\delta^{\prime}$. Then $\delta \in(0,1)$ and $m(j)>(1-\delta) l(j), j \geqslant j_{1}$. Hence,

$$
\bar{n}(\Lambda) \leqslant \frac{\gamma}{\alpha}+\varlimsup_{j \rightarrow \infty} \frac{n\left(p(j) \alpha, \Lambda^{0}\right)-n\left((1-\delta) p(j) \alpha, \Lambda^{0}\right)}{p(j) \alpha} .
$$

Since the sequence $\Lambda^{0}$ have the density $\tau_{0}$, then

$$
\begin{gathered}
\varlimsup_{j \rightarrow \infty} \frac{n\left(p(j) \alpha, \Lambda^{0}\right)-n\left((1-\delta) p(j) \alpha, \Lambda^{0}\right)}{p(j) \alpha}=\lim _{j \rightarrow \infty} \frac{n\left(p(j) \alpha, \Lambda^{0}\right)-\left((1-\delta) p(j) \alpha, \Lambda^{0}\right)}{p(j) \alpha} \\
=\lim _{j \rightarrow \infty} \frac{n\left(p(j) \alpha, \Lambda^{0}\right)}{p(j) \alpha}-\lim _{j \rightarrow \infty} \frac{n\left((1-\delta) p(j) \alpha, \Lambda^{0}\right)}{p(j) \alpha}=\tau_{0}-(1-\delta) \tau_{0}=\delta \tau_{0} .
\end{gathered}
$$

Thus,

$$
\bar{n}(\Lambda) \leqslant \frac{\gamma}{\alpha}+\delta \tau_{0}=\tau \gamma+\left(1-\gamma+\delta^{\prime}\right) \tau_{0}
$$

Since $\delta^{\prime}>0$ can be arbitrarily small, then $\bar{n}(\Lambda) \leqslant \tau \gamma+(1-\gamma) \tau_{0}$. By the inequalities $\tau_{0}<\tau$ and $\underline{n}(\Lambda) \geqslant \tau$ we obtain: $\bar{n}(\Lambda)=\tau$ and $\gamma=1$. In particular, it means that the sequence $\Lambda$ has the density $\tau$. Then

$$
n\left(\Lambda^{1}\right)=\lim _{r \rightarrow \infty} \frac{n\left(r, \Lambda^{1}\right)}{r}=\lim _{r \rightarrow \infty} \frac{n(r, \Lambda)-n\left(r, \Lambda^{0}\right)}{r}=\lim _{r \rightarrow \infty} \frac{n(r, \Lambda)}{r}-\lim _{r \rightarrow \infty} \frac{n\left(r, \Lambda^{0}\right)}{r}=\tau-\tau_{0},
$$


that is, $\Lambda^{1}$ has the density $\tau-\tau_{0}$.

It remains to prove inequalities (2.2). By construction, each semi-interval $[p \alpha,(p+1) \alpha)$, $p=0,1, \ldots$, contains at most one element of the sequence $\left\{\left|\lambda_{j}^{1}\right|\right\}_{j=1}^{\infty}$. At that, if the semiinterval $[p \alpha,(p+1) \alpha), p=0,1, \ldots$, contains the number $\left|\lambda_{j}^{1}\right|$, it lies in interval (2.3). Therefore, $\left|\lambda_{j+1}^{1}\right|-\left|\lambda_{j}^{1}\right| \geqslant \alpha-\gamma=1 / \tau-\gamma \geqslant 1 / \tau-\delta, j \geqslant 1$, that is, the right inequality in (2.2) holds true.

Let us prove the left one. We have

$$
\begin{aligned}
n((p+1) \alpha, \Lambda)-n\left(p_{0} \alpha, \Lambda\right)= & n\left((p+1) \alpha, \Lambda^{1}\right)-n\left(p_{0} \alpha, \Lambda^{1}\right) \\
& +n\left((p+1) \alpha, \Lambda^{0}\right)-n\left(p_{0} \alpha, \Lambda^{0}\right) \\
= & n\left((p+1) \alpha, \Lambda^{1}\right)-n\left(p \alpha, \Lambda^{1}\right) \\
& +n\left(p \alpha, \Lambda^{1}\right)-n\left(p_{0} \alpha, \Lambda^{1}\right)+n\left((p+1) \alpha, \Lambda^{0}\right)-n\left(p_{0} \alpha, \Lambda^{0}\right) \\
= & n\left((p+1) \alpha, \Lambda^{1}\right)-n\left(p \alpha, \Lambda^{1}\right)+N_{p-1} \\
& +n\left((p+1) \alpha, \Lambda^{0}\right)-n\left(p_{0} \alpha, \Lambda^{0}\right) .
\end{aligned}
$$

Moreover,

$$
\begin{aligned}
n((p+1) \alpha, \Lambda)-n\left(p_{0} \alpha, \Lambda\right)= & n(p \alpha, \Lambda)-n\left(p_{0} \alpha, \Lambda\right)+n\left((p+1) \alpha, \Lambda^{1}\right)-n\left(p \alpha, \Lambda^{1}\right) \\
& +n\left((p+1) \alpha, \Lambda^{0}\right)-n\left(p_{0} \alpha, \Lambda^{0}\right) .
\end{aligned}
$$

Assume that the semi-interval $[p \alpha,(p+1) \alpha)$ contains at the same time some number $\left|\lambda_{j}^{1}\right|$ and at least one element of the sequence $\left\{\left|\lambda_{k}^{0}\right|\right\}_{k=1}^{\infty}$. Then

$$
n\left((p+1) \alpha, \Lambda^{1}\right)-n\left(p \alpha, \Lambda^{1}\right)=1, \quad n\left((p+1) \alpha, \Lambda^{0}\right) n\left(p_{0} \alpha, \Lambda^{0}\right) \geqslant 1 .
$$

In view of (2.8) and the left inequality in (2.6) we obtain

$$
n((p+1) \alpha, \Lambda)-n\left(p_{0} \alpha, \Lambda\right) \geqslant n(p \alpha, \Lambda)-n\left(p_{0} \alpha, \Lambda\right)+2 \geqslant p-p_{0}+2>p+1-p_{0} .
$$

This is why by the right inequality in (2.6) we have

$$
n((p+1) \alpha, \Lambda)-n\left(p_{0} \alpha, \Lambda\right) \leqslant N_{p-1}+n\left((p+1) \alpha, \Lambda^{0}\right)-n\left(p_{0} \alpha, \Lambda^{0}\right) .
$$

Together with (2.7) this gives the identity

$$
n\left((p+1) \alpha, \Lambda^{1}\right)-n\left(p \alpha, \Lambda^{1}\right)=0,
$$

which means that the semi-interval $[p \alpha,(p+1) \alpha)$ contains no elements of the sequence $\left\{\left|\lambda_{j}^{1}\right|\right\}_{j=1}^{\infty}$. This contradicts the assumptions.

Thus, if the semi-interval $[p \alpha,(p+1) \alpha)$ contains some number $\left|\lambda_{j}^{1}\right|$, it contains no elements of the sequence $\left\{\left|\lambda_{k}^{0}\right|\right\}_{k=1}^{\infty}$. By construction, $\left|\lambda_{j}^{1}\right|$ belongs to interval (2.3). Therefore,

$$
|| \lambda_{j}^{1}|-| \lambda_{k}^{0}|| \geqslant \frac{\alpha}{2}-\frac{\gamma}{2}=\frac{1}{2 \tau}-\frac{\gamma}{2} \geqslant \frac{1}{2 \tau}-\frac{\delta}{2}, \quad k \geqslant 1 .
$$

This gives the first inequality in (2.2). The proof is complete.

Lemma 2.3. Let $\delta>0$ and $\varphi_{1} \in[-2 \pi, 0), \varphi_{1}<\varphi_{2}<\cdots<\varphi_{n}<\varphi_{n+1}=\varphi_{1}+2 \pi$, $\tau_{1}, \ldots, \tau_{n} \geqslant 0$ and $\tau=\tau_{1}+\cdots+\tau_{n}>0$. There exists a sequence $\Lambda=\left\{\lambda_{k}\right\}_{k=1}^{\infty}$ such that $\Lambda \subseteq \Lambda_{\mathbb{Z}}, n\left(\Lambda\left(\varphi_{s}, \varphi_{s+1}\right]\right)=\tau_{s}, s=\overline{1, n}$, and

$$
\left|\lambda_{k+1}\right|-\left|\lambda_{k}\right| \geqslant \frac{1}{2 \tau}-\delta, \quad k \geqslant 1 \text {. }
$$

Proof. We let $\tau_{0}=0$ and $\Lambda_{0}=\emptyset$. We seek a sequence $\Lambda \subseteq \Lambda_{\mathbb{Z}}$ as the union $\Lambda_{1} \bigcup \ldots \bigcup \Lambda_{n}$. By induction, we construct sequences $\Lambda_{s} \subseteq \Lambda_{\mathbb{Z}}\left(\varphi_{s}, \varphi_{s+1}\right), s=\overline{1, n}$, satisfying the following conditions: $\Lambda_{s}=\left\{\left|\lambda_{k}^{s}\right|\right\}_{k=1}^{\infty}$ has the density $\tau_{s}$ and as $\tau_{s}>0$, the inequalities

$$
|| \lambda_{l}^{s}|-| \lambda_{k}^{j}|| \geqslant \frac{1}{2 \widetilde{\tau}_{s}}-\frac{\delta}{2}, \quad k, l \geqslant 1, j=\overline{1, s-1}, \quad\left|\lambda_{k+1}^{s}\right|-\left|\lambda_{k}^{s}\right| \geqslant \frac{1}{\widetilde{\tau}_{s}}-\delta, \quad k \geqslant 1,
$$


hold true, where $\widetilde{\tau}_{s}=\tau_{1}+\cdots+\tau_{s}$, if $\Lambda_{0} \cup \ldots \cup \Lambda_{s-1}=\emptyset$, then the first inequality in (2.10) is omitted. If $\tau_{s}=0$, then $\Lambda_{s}=\emptyset$.

Let $s=1$. If $\tau_{1}=0$, we let $\Lambda_{1}=\emptyset$. Otherwise by Lemma 2.2 there exists a sequence $\Lambda_{1}=\left\{\left|\lambda_{k}^{1}\right|\right\}_{k=1}^{\infty} \subseteq \Lambda_{\mathbb{Z}}\left(\varphi_{1}, \varphi_{2}\right)$ having the density $\widetilde{\tau}_{1}-\tau_{0}=\tau_{1}$ such that (2.10) holds true.

We assume that the sought sequences $\Lambda_{j}, j=\overline{1, s-1}$, are constructed. In Lemma 2.2, as $\Lambda_{0}$ we choose the union $\Lambda_{1} \cup \ldots \cup \Lambda_{s-1}$. Then by this lemma there exists a sequence $\Lambda_{s}=$ $\left\{\lambda_{k}^{s}\right\}_{k=1}^{\infty} \subseteq \Lambda_{\mathbb{Z}}\left(\varphi_{j}, \varphi_{j+1}\right)$ having the density $\tau_{s}=\widetilde{\tau}_{s}-\widetilde{\tau}_{s-1}$ such that (2.10). Thus, we have constructed the sequence $\Lambda=\left\{\lambda_{k}\right\}_{k=1}^{\infty} \subseteq \Lambda_{\mathbb{Z}}$, for which $n\left(\Lambda\left(\varphi_{s}, \varphi_{s+1}\right]\right)=n\left(\Lambda_{s}\right)=\tau_{s}, s=\overline{1, n}$. Since $\tau \geqslant \widetilde{\tau}_{s}, s=\overline{1, n}$, it satisfies (2.9). The proof is complete.

Let us consider more precise characteristics of the sequence $\Lambda=\left\{\lambda_{k}\right\}_{k=1}^{\infty}$.

The lower and upper densities of $\Lambda$ in the angle $\Gamma\left(\varphi_{1}, \varphi_{2}\right)$ are the corresponding densities of the sequence $\Lambda\left(\varphi_{1}, \varphi_{2}\right)$.

A sequence $\Lambda$ is said to have an angular density $n\left(\Lambda, \varphi_{1}, \varphi_{2}\right)<+\infty$ (at order one) if for all admissible $\varphi_{1}, \varphi_{2}$ except probably a countable set $\Phi_{\Lambda}$, the identity $\underline{n}\left(\Lambda\left(\varphi_{1}, \varphi_{2}\right)\right)=$ $\bar{n}\left(\Lambda\left(\varphi_{1}, \varphi_{2}\right)\right)=n\left(\Lambda, \varphi_{1}, \varphi_{2}\right)$ holds true [3, Ch. II, Sect. 1]. We have $\widetilde{\varphi} \in \Phi_{\Lambda} \backslash\{-2 \pi\}$ if and only if $\inf _{\varphi>0} \bar{n}(\Lambda(\widetilde{\varphi}-\varphi, \widetilde{\varphi}+\varphi))>0$, where $\varphi$ is sufficiently small. The number $-2 \pi$ belongs or does not to $\Phi_{\Lambda}$ simultaneously with $\widetilde{\varphi}=0$.

By the symbol $\Sigma$ we denote the class of non-decaying in $[-2 \pi, 2 \pi]$ functions $\omega(\varphi)$ possessing the following properties: $\omega(0)=0, \omega$ is right-continuous, $\omega(\varphi)=\omega(\varphi-2 \pi)-\omega(-2 \pi), \varphi \in$ $[0,2 \pi)$. By $\Phi(\omega)$ we denote the set of the discontinuity points of the function $\omega$.

Let $\Lambda$ have an angular density. Then it determines uniquely the function $\omega_{\Lambda} \in \Sigma$ by the rule: for $\varphi_{1}, \varphi_{2} \in(-2 \pi, 0) \backslash \Phi_{\Lambda}, \varphi \in\left(\varphi_{1}, \varphi_{1}+2 \pi\right) \backslash \Phi_{\Lambda}$

$$
\omega_{\Lambda}\left(\varphi_{1}\right)=-\lim _{\varphi_{2} \rightarrow 0} n\left(\Lambda, \varphi_{1}, \varphi_{2}\right), \quad \omega_{\Lambda}(\varphi)=n\left(\Lambda, \varphi_{1}, \varphi\right)+\omega_{\Lambda}\left(\varphi_{1}\right) .
$$

More precisely, $\omega_{\Lambda}$ is continued uniquely to a function in the class $\Sigma$, and the continuation is independent of $\varphi_{1}$. It is easy to see that the sets $\Phi_{\Lambda}$ and $\Phi\left(\omega_{\Lambda}\right)$ coincide. The definition of $\omega_{\Lambda}$ implies the identity $n\left(\Lambda, \varphi_{1}, \varphi_{2}\right)=\omega_{\Lambda}\left(\varphi_{2}\right)-\omega_{\Lambda}\left(\varphi_{1}\right)$ for all admissible $\varphi_{1}, \varphi_{2} \notin \Phi_{\Lambda}=\Phi\left(\omega_{\Lambda}\right)$. At that, $n(\Lambda)=\omega_{\Lambda}(\varphi+2 \pi)-\omega_{\Lambda}(\varphi), \varphi \in[-2 \pi, 0)$. We shall say that the sequence $\Lambda$ has an angular density $\omega \in \Sigma$ if it has an angular density and $\omega_{\Lambda}=\omega$.

Lemma 2.4. Let $\omega \in \Sigma$ and $\Lambda$ are such that for some $\varphi_{1} \in(-2 \pi, 0) \backslash \Phi(\omega)$ and all $\varphi, \psi \notin$ $\Phi(\omega)$ obeying the condition $\varphi_{1} \leqslant \varphi<\psi \leqslant \varphi_{1}+2 \pi$ the sequence $\Lambda(\varphi, \psi]$ has a density and $n(\Lambda(\varphi, \psi])=\omega(\psi)-\omega(\varphi)$. Then $\Lambda$ has an angular density $\omega$.

Proof. Let $\widetilde{\varphi}_{1}, \widetilde{\varphi}_{2} \notin \Phi_{\Lambda}$ be admissible. Subject to the location of the points $\widetilde{\varphi}_{1}, \widetilde{\varphi}_{2}$ in the segment $[-2 \pi, 2 \pi]$, several situations are possible. We shall study two of them, the other can be considered in the same way.

1. $\widetilde{\varphi}_{2}=\varphi_{1}$. In this case $\Lambda\left(\widetilde{\varphi}_{1}, \widetilde{\varphi}_{2}\right)=\Lambda\left(\widetilde{\varphi}_{1}+2 \pi, \varphi_{1}+2 \pi\right) \subseteq \Lambda\left(\widetilde{\varphi}_{1}+2 \pi, \varphi_{1}+2 \pi\right]$. By the assumption, $\left(\varphi_{1}+2 \pi\right) \notin \Phi(\omega)$. Let us show that $\left(\widetilde{\varphi}_{1}+2 \pi\right) \notin \Phi(\omega)$. Since $\widetilde{\varphi}_{1} \notin \Phi_{\Lambda}$, then

$$
\inf _{\varphi>0} \bar{n}\left(\Lambda\left(\widetilde{\varphi}_{1}+2 \pi-\varphi, \widetilde{\varphi}_{1}+2 \pi+\varphi\right)\right)=\inf _{\varphi>0} \bar{n}\left(\Lambda\left(\widetilde{\varphi}_{1}-\varphi, \widetilde{\varphi}_{1}+\varphi\right)\right)=0 .
$$

Let $\psi_{l} \rightarrow 0$ be such that $\left(\widetilde{\varphi}_{1}+2 \pi \pm \psi_{l}\right) \notin \Phi(\omega), l \geqslant 1$. Then by the assumption we have

$$
\begin{aligned}
\omega\left(\widetilde{\varphi}_{1}+2 \pi+\psi_{l}\right)-\omega\left(\widetilde{\varphi}_{1}+2 \pi-\psi_{l}\right) & \left.=n\left(\Lambda\left(\widetilde{\varphi}_{1}\right)+2 \pi-\psi_{l}, \widetilde{\varphi}_{1}+2 \pi+\psi_{l}\right]\right) \\
& \leqslant \bar{n}\left(\Lambda\left(\widetilde{\varphi}_{1}+2 \pi-2 \psi_{l}, \widetilde{\varphi}_{1}+2 \pi+2 \psi_{l}\right)\right) \rightarrow 0, \quad l \rightarrow \infty .
\end{aligned}
$$

Since $\omega$ does not decay, this implies the desired statement. Then by the definition of the upper density and in view of Lemma 2.1 we obtain

$$
\bar{n}\left(\Lambda\left(\widetilde{\varphi}_{1}, \widetilde{\varphi}_{2}\right)\right) \leqslant \bar{n}\left(\Lambda\left(\widetilde{\varphi}_{1}+2 \pi, \widetilde{\varphi}_{2}+2 \pi\right]\right)=\omega\left(\varphi_{1}+2 \pi\right)-\omega\left(\widetilde{\varphi}_{1}+2 \pi\right) .
$$


On other hand, similar arguments yield the inequality

$$
\underline{n}\left(\Lambda\left(\widetilde{\varphi}_{1}, \widetilde{\varphi}_{2}\right)\right) \geqslant \underline{n}\left(\Lambda\left(\widetilde{\varphi}_{1}+2 \pi, \varphi_{1}+2 \pi-\psi_{l}\right]\right)=\omega\left(\varphi_{1}+2 \pi-\psi_{l}\right)-\omega\left(\widetilde{\varphi}_{1}+2 \pi\right), \quad l \geqslant 1,
$$

where $0<\psi_{l} \rightarrow 0$ and $\left(\varphi_{1}+2 \pi-\psi_{l}\right) \notin \Phi(\omega)$. Hence, employing the continuity of $\omega$ at the point $\varphi_{1}+2 \pi$ and the previous inequality we find that $\Lambda\left(\widetilde{\varphi}_{1}, \widetilde{\varphi}_{2}\right)$ has the density $\omega\left(\varphi_{1}+2 \pi\right)-$ $\omega\left(\widetilde{\varphi}_{1}+2 \pi\right)==\omega\left(\varphi_{1}\right)-\omega\left(\widetilde{\varphi}_{1}\right)=\omega\left(\widetilde{\varphi}_{2}\right)-\omega\left(\widetilde{\varphi}_{1}\right)$.

2. $\widetilde{\varphi}_{2}>\varphi_{1}, \widetilde{\varphi}_{1}<\varphi_{1}$. As above, one can show that $\widetilde{\varphi}_{2}, \widetilde{\varphi}_{1}+2 \pi \notin \Phi(\omega)$ and $\Lambda\left(\varphi_{1}, \widetilde{\varphi}_{2}\right)$ has density $\omega\left(\widetilde{\varphi}_{2}\right)-\omega\left(\varphi_{1}\right)$. In view of the assumption we have

$$
\begin{aligned}
n\left(\Lambda\left(\widetilde{\varphi}_{1}, \widetilde{\varphi}_{2}\right)\right) & =n\left(\Lambda\left(\widetilde{\varphi}_{1}, \varphi_{1}\right]\right)+n\left(\Lambda\left(\varphi_{1}, \widetilde{\varphi}_{2}\right)\right)=n\left(\Lambda\left(\widetilde{\varphi}_{1}+2 \pi, \varphi_{1}+2 \pi\right]\right)+n\left(\Lambda\left(\varphi_{1}, \widetilde{\varphi}_{2}\right)\right) \\
& =\omega\left(\varphi_{1}+2 \pi\right)-\omega\left(\widetilde{\varphi}_{1}+2 \pi\right)+\omega\left(\widetilde{\varphi}_{2}\right)-\omega\left(\varphi_{1}\right)=\omega\left(\widetilde{\varphi}_{2}\right)-\omega\left(\widetilde{\varphi}_{1}\right) .
\end{aligned}
$$

By the continuity of $\omega$ at point $\varphi_{1}$ it implies that $\varphi_{1} \notin \Phi_{\Lambda}$.

Thus, $\Lambda$ has the angular density $n\left(\Lambda, \widetilde{\varphi}_{1}, \widetilde{\varphi}_{2}\right)=\omega\left(\widetilde{\varphi}_{2}\right)-\omega\left(\widetilde{\varphi}_{1}\right)$. It remains to show $\omega_{\Lambda}=\omega$. In view of the above fact this is implied immediately by the definition of $\omega_{\Lambda}$, the left-continuity of function $\omega$ and identity $\omega(0)=0$. The proof is complete.

The proof of the following statement is based on the method used in the proof of Theorem 2.1 in work [6].

Theorem 1. Let $\omega \in \Sigma$ and $\delta>0$. There is a sequence $\Lambda=\left\{\lambda_{k}\right\}_{k=1}^{\infty} \subseteq \Lambda_{\mathbb{Z}}$ with an angular density $\omega$ such that

$$
\left|\lambda_{k+1}\right|-\left|\lambda_{k}\right| \geqslant \frac{1}{2\left(\omega_{\Lambda}\left(\varphi_{1}+2 \pi\right)-\omega_{\Lambda}\left(\varphi_{1}\right)\right)}-\delta, \quad k \geqslant 1,
$$

where $\varphi_{1} \in(-2 \pi, 0) \backslash \Phi(\omega)$ is chosen arbitrarily.

Proof. First of all, let us construct a special set of sequences $\Lambda^{j} \subseteq \Lambda_{\mathbb{Z}}, j \geqslant 1$. Then we shall "glue" sequence $\Lambda$ with the desired angular density from the parts of $\Lambda^{j}$. Let $\varphi_{1} \in$ $(-2 \pi, 0) \backslash \Phi(\omega)$. For each $j \geqslant 1$ we fix a set of numbers $\varphi_{s}^{j} \notin \Phi(\omega), s=\overline{1, s(j)}$ such that $\varphi_{1}^{j}=\varphi_{1}, \varphi_{1}^{j}<\varphi_{2}^{j}<\cdots<\varphi_{s(j)}^{j}<\varphi_{1}+2 \pi=\varphi_{s(j)+1}^{j}$ and $\varphi_{s+1}^{j}-\varphi_{s}^{j}<1 / j, s=\overline{1, s(j)}$. By Lemma 2.3 , for each $j \geqslant 1$ there exists a sequence $\Lambda^{j}=\left\{\lambda_{k}^{j}\right\}_{k=1}^{\infty} \subseteq \Lambda_{\mathbb{Z}}$ such that

$$
\begin{gathered}
n\left(\Lambda^{j}\left(\varphi_{s}^{j}, \varphi_{s+1}^{j}\right]\right)=\tau_{s}^{j}=\omega\left(\varphi_{s+1}^{j}\right)-\omega\left(\varphi_{s}^{j}\right), \quad s=\overline{1, s(j)}, \quad j \geqslant 1, \\
\left|\lambda_{k+1}^{j}\right|-\left|\lambda_{k}^{j}\right| \geqslant \alpha=\frac{1}{2 \tau}-\delta, \quad k \geqslant 1,
\end{gathered}
$$

where $\tau=\tau_{1}^{j}+\cdots+\tau_{s(j)}^{j}$. By (2.12) we obtain $n\left(\Lambda^{j}\right)=\tau=\omega_{\Lambda}\left(\varphi_{1}+2 \pi\right)-\omega_{\Lambda}\left(\varphi_{1}\right)$.

Let $j \geqslant 1$. By $(2.12)$, there exists a number $R_{j}>0$ satisfying the condition

$$
\left|\frac{n\left(r, \Lambda^{j}\left(\varphi_{s}^{j}, \varphi_{s+1}^{j}\right]\right)}{r}-\left(\omega\left(\varphi_{s+1}^{j}\right)-\omega\left(\varphi_{s}^{j}\right)\right)\right|<\frac{1}{j s(j)}, \quad s=\overline{1, s(j)}, \quad r \geqslant R_{j} .
$$

We can assume that

$$
R_{j+1} \geqslant 2 R_{j}, \quad R_{j+1}-\alpha>R_{j}, \quad j \geqslant 1 .
$$

Let $\Lambda^{j, j}$ be the set of all elements in the sequence $\Lambda^{j}$ located in the ring $\left\{\lambda \in \mathbb{C}: R_{j} \leqslant|\lambda|<R_{j+1}-\alpha\right\}$. We let $\Lambda=\bigcup_{j \geqslant 1} \Lambda^{j, j}$. By construction, $\Lambda=\left\{\lambda_{k}\right\}_{k=1}^{\infty} \subseteq \Lambda_{\mathbb{Z}}$. By (2.13), condition (2.11) holds for all points in set $\Lambda^{j, j}, j \geqslant 1$. Then by construction $\left|\lambda_{k}^{j}\right|-\left|\lambda_{n}^{l}\right| \geqslant \alpha$ if $j \neq l$ and $\lambda_{k}^{j} \in \Lambda^{j, j}$, $\lambda_{n}^{l} \in \Lambda^{l, l}$. It follows that condition (2.11) holds true for the sequence $\Lambda$. It remains to show that $\Lambda$ has the angular density $\omega$. 
We fix numbers $\varphi, \psi \notin \Phi(\omega)$ such that $\varphi_{1} \leqslant \varphi<\psi \leqslant \varphi_{1}+2 \pi$. Let us prove the identity $n(\Lambda(\varphi, \psi])=\omega(\psi)-\omega(\varphi)$. Let $r>0$ and an index $j(r)$ be such that $R_{j(r)-1}<r \leqslant R_{j(r)}$. By construction, for each index $j_{0}$ and all $r>R_{j_{0}}$ we have

$$
\begin{aligned}
n(r, \Lambda(\varphi, \psi])= & n\left(R_{j_{0}}, \Lambda(\varphi, \psi]\right)+\sum_{j=j_{0}}^{j(r)-2}\left(n\left(R_{j+1}-\alpha, \Lambda^{j}(\varphi, \psi]\right)-n\left(R_{j}, \Lambda^{j}(\varphi, \psi]\right)\right) \\
& +n\left(t(r), \Lambda^{j(r)-1}(\varphi, \psi]\right)-n\left(R_{j(r)-1}, \Lambda^{j(r)-1}(\varphi, \psi]\right),
\end{aligned}
$$

where $t(r)=\min \left\{r, R_{j(r)}-\alpha\right\}$.

For each $j \geqslant 1$ there exist indices $1 \leqslant i(j) \leqslant l(j) \leqslant s(j)$ such that the embeddings

$$
\bigcup_{s=i(j)+1}^{l(j)-1} \Gamma\left(\varphi_{s}^{j}, \varphi_{s+1}^{j}\right] \subseteq \Gamma(\varphi, \psi] \subseteq \bigcup_{s=i(j)}^{l(j)} \Gamma\left(\varphi_{s}^{j}, \varphi_{s+1}^{j}\right]
$$

hold true. For finitely many indices $j$ we can have $i(j)+1>l(j)-1$; in this case the left embedding is absent. Then we obtain

$$
\begin{gathered}
\sum_{s=i(j)+1}^{l(j)-1}\left(n\left(r, \Lambda^{j}\left(\varphi_{s}^{j}, \varphi_{s+1}^{j}\right]\right)-n\left(\widetilde{r}, \Lambda^{j}\left(\varphi_{s}^{j}, \varphi_{s+1}^{j}\right]\right)\right) \leqslant n\left(r, \Lambda^{j}(\varphi, \psi]\right)-n\left(\widetilde{r}, \Lambda^{j}(\varphi, \psi]\right) \\
\leqslant \sum_{s=i(j)}^{l(j)}\left(n\left(r, \Lambda^{j}\left(\varphi_{s}^{j}, \varphi_{s+1}^{j}\right]\right)-n\left(\widetilde{r}, \Lambda^{j}\left(\varphi_{s}^{j}, \varphi_{s+1}^{j}\right]\right)\right), \quad 0<\widetilde{r}<r .
\end{gathered}
$$

Let $1 \leqslant i \leqslant l \leqslant s(j)$ and $r>\widetilde{r} \geqslant R_{j}$. By (2.14) the inequality

$$
\left|\sum_{s=i}^{l}\left(n\left(r, \Lambda^{j}\left(\varphi_{s}^{j}, \varphi_{s+1}^{j}\right]\right)-n\left(\widetilde{r}, \Lambda^{j}\left(\varphi_{s}^{j}, \varphi_{s+1}^{j}\right]\right)\right)-(r-\widetilde{r}) \sum_{s=i}^{l}\left(\omega\left(\varphi_{s+1}^{j}\right)-\omega\left(\varphi_{s}^{j}\right)\right)\right| \leqslant \frac{2 r}{j},
$$

holds true. Therefore,

$$
\left|\sum_{s=i}^{l}\left(n\left(r, \Lambda^{j}\left(\varphi_{s}^{j}, \varphi_{s+1}^{j}\right]\right)-n\left(\widetilde{r}, \Lambda^{j}\left(\varphi_{s}^{j}, \varphi_{s+1}^{j}\right]\right)\right)-(r-\widetilde{r})\left(\omega\left(\varphi_{l}^{j}\right)-\omega\left(\varphi_{i}^{j}\right)\right)\right| \leqslant \frac{2 r}{j} .
$$

In view of (2.17) this implies:

$$
\begin{gathered}
(r-\widetilde{r})\left(\omega\left(\varphi_{l(j)-1}^{j}\right)-\omega\left(\varphi_{i(j)+1}^{j}\right)\right)-2 r / j \leqslant n\left(r, \Lambda^{j}(\varphi, \psi]\right)-n\left(\widetilde{r}, \Lambda^{j}(\varphi, \psi]\right) \\
\leqslant(r-\widetilde{r})\left(\omega\left(\varphi_{l(j)}^{j}\right)-\omega\left(\varphi_{i(j)}^{j}\right)\right)+2 r / j, \quad j \geqslant 1, \quad r>\widetilde{r} \geqslant R_{j} .
\end{gathered}
$$

Let $\varepsilon>0$. By the continuity of $\omega$ at the points $\psi$ and $\varphi$ there exists $\delta>0$ such that

$$
|\omega(\widetilde{\psi})-\omega(\widetilde{\varphi})-(\omega(\psi)-\omega(\varphi))|<\varepsilon, \quad \forall \widetilde{\psi}, \widetilde{\varphi}:|\widetilde{\psi}-\psi|<\delta,|\widetilde{\varphi}-\varphi|<\delta .
$$

We choose $j_{0} \geqslant \max \{1 / \delta, 1 / \varepsilon\}$. Then in view of (2.16), (2.18), (2.19) and (2.15) we have

$$
\begin{aligned}
n(r, \Lambda(\varphi, \psi]) \geqslant & n\left(R_{j_{0}}, \Lambda(\varphi, \psi]\right)+\sum_{j=j_{0}}^{j(r)-2}\left(\left(R_{j+1}-\alpha-R_{j}\right)\left(\omega\left(\varphi_{l(j)}^{j}\right)-\omega\left(\varphi_{i(j)}^{j}\right)\right)-\frac{2 R_{j+1}}{j}\right) \\
& +\left(t(r)-R_{j(r)-1}\right)\left(\omega\left(\varphi_{l(j(r)-1)}^{j(r)-1}\right)-\omega\left(\varphi_{i(j(r)-1)}^{j(r)-1}\right)\right)-\frac{2 t(r)}{j(r)-1} \\
\geqslant & n\left(R_{j_{0}}, \Lambda(\varphi, \psi]\right)++\sum_{j=j_{0}+1}^{j(r)-1}\left(\left(R_{j+1}-\alpha-R_{j}\right)(\omega(\psi)-\omega(\varphi)-\varepsilon)-\frac{2 R_{j+1}}{j}\right)
\end{aligned}
$$




$$
\begin{aligned}
& +\left(t(r)-R_{j(r)-1}\right)(\omega(\psi)-\omega(\varphi)-\varepsilon)-\frac{2 t(r)}{j(r)-1} \\
\geqslant & n\left(R_{j_{0}}, \Lambda(\varphi, \psi]\right)+(\omega(\psi)-\omega(\varphi)-\varepsilon)\left(\sum_{j=j_{0}}^{j(r)-2}\left(R_{j+1}-\alpha-R_{j}\right)+t(r)-R_{j(r)-1}\right) \\
& \quad-\sum_{j=j_{0}}^{j(r)-2} \frac{2 R_{j+1}}{j}-\frac{2 t(r)}{j(r)-1} \\
\geqslant & n\left(R_{j_{0}}, \Lambda(\varphi, \psi]\right)+(\omega(\psi)-\omega(\varphi)-\varepsilon)\left(r-R_{j_{0}}-\alpha j(r)\right)-6 \varepsilon r, \quad r>R_{j_{0}} .
\end{aligned}
$$

Therefore,

$$
\underline{n}(\Lambda(\varphi, \psi])=\varliminf_{r \rightarrow \infty} \frac{n(r, \Lambda(\varphi, \psi])}{r} \geqslant \omega(\psi)-\omega(\varphi)-\varepsilon-\varliminf_{r \rightarrow \infty} \frac{\alpha j(r)}{r}-6 \varepsilon .
$$

By the choice of index $j(r)$ and (2.15), the inequalities $r>R_{j(r)-1} \geqslant 2^{j(r)-2} R_{1}$ hold true. This is why $\underline{n}(\Lambda(\varphi, \psi]) \geqslant \omega(\psi)-\omega(\varphi)-7 \varepsilon$. Since $\varepsilon>0$ is arbitrary, then $\underline{n}(\Lambda(\varphi, \psi]) \geqslant \omega(\psi)-\omega(\varphi)$. In the same way we get the upper bound: $\bar{n}(\Lambda(\varphi, \psi]) \leqslant \omega(\psi)-\omega(\varphi)$. Hence, we have the identities $\underline{n}(\Lambda(\varphi, \psi])=\bar{n}(\Lambda(\varphi, \psi])=n(\Lambda(\varphi, \psi])=\omega(\psi)-\omega(\varphi)$. By Lemma 2.4 it yields that $\Lambda$ has angular density $\omega$. The proof is complete.

We recall that sequence $\Lambda=\left\{\lambda_{k}\right\}_{k=1}^{\infty}$ is called properly distributed set (see [1, Ch. II, Sect. 1]) at order one if it has an angular density and Lindelöf condition is satisfied, that is, there exists $\lim _{r \rightarrow \infty} N(r, \Lambda)$, where

$$
N(r, \Lambda)=\sum_{\left|\lambda_{k}\right|<r} \frac{1}{\lambda_{k}}
$$

In the following statements we give an answer to the question how a sequence with an angular density can be "converted" into a properly distributed set.

Let $\Lambda$ have an angular density. We shall say that $\Lambda$ is a general form sequence if there exist $\varphi_{1}, \varphi_{2}, \varphi_{3} \in[-\pi, \pi)$ such that $\varphi_{1}<\varphi_{2}<\varphi_{3}, \varphi_{2}-\varphi_{1}<\pi, \varphi_{3}-\varphi_{2}<\pi, \varphi_{1}+2 \pi-\varphi_{3}<\pi$ and

$$
n\left(\Lambda\left(\varphi_{j}-\varphi, \varphi_{j}+\varphi\right)\right)>0, \quad j=1,2,3, \quad \varphi \in(0, \pi / 2) .
$$

We observe that the function depending on $\varphi$ in the left hand side in this inequality is nondecreasing. This is it is sufficient to satisfy the inequality at some sequence $\varphi=\psi_{j, p} \rightarrow 0$.

Lemma 2.5. Let $a>1, \Lambda=\left\{\lambda_{k}\right\}_{k=1}^{\infty}$ and $\mathbb{C} \ni \gamma_{m} \rightarrow 0, m \rightarrow \infty$. Assume that $\Lambda$ is a general form sequence. Then there exists a sequence of zero density $T \subset \Lambda$ such that

$$
\sum_{m=1}^{l} \gamma_{m}-N\left(a^{l+1}, T\right) \rightarrow 0, \quad l \rightarrow \infty .
$$

Proof. Let $\varphi_{1}, \varphi_{2}, \varphi_{3}$ be the numbers involved in the definition of the general form sequence. We let

$$
\varphi_{0}=4^{-1} \min \left\{\pi-\left(\varphi_{2}-\varphi_{1}\right) ; \pi-\left(\varphi_{3}-\varphi_{2}\right) ; \pi-\left(\varphi_{1}+2 \pi-\varphi_{3}\right)\right\}<\pi / 4 .
$$

Let us observe an important property of numbers $\varphi_{0}, \varphi_{1}, \varphi_{2}, \varphi_{3}$. For an arbitrary straight line passing through the origin and for each of two half-planes created by this line, there exists $j=1,2,3$ such that the angle $\Gamma_{j}=\Gamma\left(\varphi_{j}-2 \varphi_{0}, \varphi_{j}+2 \varphi_{0}\right)$ lies in this half-plane.

We seek the set $T$ as $T=\bigcup_{m=1}^{\infty} T_{m}$, where $T_{m}=\left\{t_{l}\right\}_{l=\widetilde{p}(m-1)+1}^{\widetilde{p}(m)}=\left\{\lambda_{k(m, p)}\right\}_{p=1}^{p(m)}$ is a some subset of $\Lambda$ lying in the ring $K(m)=\left\{\xi: a^{m}<|\xi| \leqslant a^{m+1}\right\}$. It is possible that $T_{m}=\emptyset$ (that is, $p(m)=0, \widetilde{p}(m)=\widetilde{p}(m-1))$ for some $m$. 
We let $\widetilde{p}(0)=p(0)=0, \gamma_{0}(0)=0, \gamma_{m}(0)=\gamma_{m-1}(p(m-1))+\gamma_{m}, m \geqslant 1$,

$$
\gamma_{m}(p)=\gamma_{m}(0)-\sum_{\mu=\widetilde{p}(m-1)+1}^{\widetilde{p}(m-1)+p} \frac{1}{t_{\mu}}, \quad p=\overline{1, p(m)} .
$$

Let $\widetilde{\Gamma}_{j}=\Gamma\left(\varphi_{j}-\varphi_{0}, \varphi_{j}+\varphi_{0}\right), j=1,2,3, \Pi(\varphi)=\left\{\xi \in \mathbb{C}: \operatorname{Re}\left(\xi e^{i \varphi}\right\}>0, \varphi(m, p)\right.$ be the argument of number $\gamma_{m}(p)$ and $j(m, p), m \neq 0$, be the index such that $\Gamma_{j(m, p)} \subset \Pi(\varphi(m, p))$.

For each $m \geqslant 1$ we choose a set $T_{m}=\left\{t_{l}\right\}_{l=\widetilde{p}(m-1)+1}^{\widetilde{p}(m-1)+p(m)}$ such that

1) $p(m)$ is the minimal non-negative integer number for which either $\left|\gamma_{m}(p(m))\right| \leqslant$ $\left(a^{m} \sin \varphi_{0}\right)^{-1}$ or the set $K(m) \bigcap \Gamma_{j(m, p(m))}$ contains no points in the sequence $\Lambda \backslash T_{m}$.

2) For each $p=\overline{1, p(m)}$, the number $t_{\widetilde{p}(m-1)+p}$ is an arbitrary element $\lambda_{k(m, p)} \in \Lambda \backslash T_{m, p-1}$, where $T_{m, 0}=\emptyset$ and $T_{m, p-1}=\left\{\lambda_{k(m, s)}\right\}_{s=1}^{p-1}, p>1$, and this element belongs to the intersection $K(m) \cap \widetilde{\Gamma}_{j(m, p-1)}$.

Thus, the set $T=\bigcup_{m=1}^{\infty} T_{m}$ is well-defined. Let us find the upper bound for the indices $p(m)>0$. First of all, let us prove the inequality

$$
\left|\gamma_{m}(p)\right| \leqslant\left|\gamma_{m}(p-1)\right|-2^{-1} a^{-m-1} \sin \varphi_{0}, \quad p=\overline{1, p(m)}
$$

In accordance with $(2.20)$ we have $\gamma_{m}(p)=\gamma_{m}(p-1)-\left(\lambda_{k(m, p)}\right)^{-1}$. Then by the cosine theorem

$$
\left|\gamma_{m}(p)\right|^{2}=\left|\gamma_{m}(p-1)\right|^{2}+\left|\lambda_{k(m, p)}\right|^{-2}-2\left|\gamma_{m}(p-1)\right|\left|\lambda_{k(m, p)}\right|^{-1} \cos \alpha,
$$

where $\alpha$ is an angle between the vectors $\gamma_{m}(p-1)$ and $\left(\lambda_{k(m, p)}\right)^{-1}$, which does not exceed $\pi / 2-\varphi_{0}$ (such angle exists since $\left.\lambda_{k(m, p)} \in \widetilde{\Gamma}_{j(m, p-1)}\right)$. Since $\lambda_{k(m, p)} \in K(m)$ and by 1) the inequality $\left|\gamma_{m}(p-1)\right|>\left(a^{m} \sin \varphi_{0}\right)^{-1}$ holds true, then

$$
\begin{aligned}
\left|\gamma_{m}(p-1)\right|^{2}-\left|\gamma_{m}(p)\right|^{2} & \geqslant 2\left|\gamma_{m}(p-1)\right|\left|\lambda_{k(m, p)}\right|^{-1} \sin \varphi_{0}-\left|\lambda_{k(m, p)}\right|^{-2} \\
& =\left|\gamma_{m}(p-1)\right|\left|\lambda_{k(m, p)}\right|^{-1}\left(2 \sin \varphi_{0}-\left(\left|\lambda_{k(m, p)}\right|\left|\gamma_{m}(p-1)\right|\right)^{-1}\right) \\
& \geqslant\left|\gamma_{m}(p-1)\right|\left|\lambda_{k(m, p)}\right|^{-1}\left(2 \sin \varphi_{0}-\sin \varphi_{0}\right) \geqslant\left|\gamma_{m}(p-1)\right| a^{-m-1} \sin \varphi_{0} .
\end{aligned}
$$

In particular, $\left|\gamma_{m}(p-1)\right|>\left|\gamma_{m}(p)\right|$. Therefore,

$$
\begin{aligned}
2\left|\gamma_{m}(p-1)\right|\left(\left|\gamma_{m}(p-1)\right|-\left|\gamma_{m}(p)\right|\right) & \geqslant\left(\left|\gamma_{m}(p-1)\right|+\left|\gamma_{m}(p)\right|\right)\left(\left|\gamma_{m}(p-1)\right|-\left|\gamma_{m}(p)\right|\right) \\
& \geqslant\left|\gamma_{m}(p-1)\right| a^{-m-1} \sin \varphi_{0} .
\end{aligned}
$$

It follows inequality (2.21). Applying it $p(m)$ times, we get

$$
0 \leqslant\left|\gamma_{m}(p(m))\right| \leqslant\left|\gamma_{m}(0)\right|-2^{-1} a^{-m-1} p(m) \sin \varphi_{0}, \quad m \geqslant 1 .
$$

For $p(m)=0$ this inequality is trivial. This is why

$$
p(m) \leqslant 2 a^{m+1}\left(\sin \varphi_{0}\right)^{-1}\left|\gamma_{m}(0)\right|, \quad m \geqslant 1 .
$$

Let us show that

$$
\gamma_{l}(p(l))=\sum_{m=1}^{l} \gamma_{m}-N\left(a^{l+1}, T\right) \rightarrow 0, \quad l \rightarrow \infty .
$$

By the assumption, $n\left(\Lambda\left(\varphi_{j}-\varphi_{0}, \varphi_{j}+\varphi_{0}\right)\right)>0, j=1,2,3$. Then

$$
\begin{aligned}
\lim _{m \rightarrow \infty} & \frac{n\left(a^{m+1}, \Lambda\left(\varphi_{j}-\varphi_{0}, \varphi_{j}+\varphi_{0}\right)\right)-n\left(a^{m}, \Lambda\left(\varphi_{j}-\varphi_{0}, \varphi_{j}+\varphi_{0}\right)\right)}{a^{m+1}} \\
\quad=n\left(\Lambda\left(\varphi_{j}-\varphi_{0}, \varphi_{j}+\varphi_{0}\right)\right)-a^{-1} n\left(\Lambda\left(\varphi_{j}-\varphi_{0}, \varphi_{j}+\varphi_{0}\right)\right)>0, \quad j=1,2,3 . &
\end{aligned}
$$

Therefore, there exist a number $\tau>0$ and an index $m_{0}$ such that

$$
n\left(a^{m+1}, \Lambda\left(\varphi_{j}-\varphi_{0}, \varphi_{j}+\varphi_{0}\right)\right)-n\left(a^{m}, \Lambda\left(\varphi_{j}-\varphi_{0}, \varphi_{j}+\varphi_{0}\right)\right) \geqslant a^{m+1}, \quad j=1,2,3, \quad m \geqslant m_{0} .
$$


In view of (2.22) and 1$), 2)$ we obtain

$$
\left|\gamma_{m}(p(m))\right| \leqslant \max \left\{\left(a^{m} \sin \varphi_{0}\right)^{-1},\left|\gamma_{m}(0)\right|-2^{-1} \tau \sin \varphi_{0}\right\}, \quad m \geqslant m_{0} .
$$

In accordance with the assumption of the lemma we can assume that

$$
\left|\gamma_{m}\right|+\left(a^{m-1} \sin \varphi_{0}\right)^{-1} \leqslant 4^{-1} \tau \sin \varphi_{0}, \quad m \geqslant m_{0} .
$$

Assume that $\left|\gamma_{m}(p(m))\right|>\left(a^{m} \sin \varphi_{0}\right)^{-1}$ for all $m \geqslant m_{0}$. Then by (2.25), (2.26) and the definition of $\gamma_{m}(0)$ we have

$$
\begin{aligned}
\left|\gamma_{l}(p(l))\right| & \leqslant\left|\gamma_{l}(0)\right|-2^{-1} \tau \sin \varphi_{0} \leqslant\left|\gamma_{l-1}(p(l-1))\right|+\left|\gamma_{l}\right|-2^{-1} \tau \sin \varphi_{0} \\
& \leqslant\left|\gamma_{l-1}(p(l-1))\right|-4^{-1} \tau \sin \varphi_{0} \leqslant \cdots \leqslant\left|\gamma_{m_{0}}\left(p\left(m_{0}\right)\right)\right|-4^{-1} \tau\left(l-m_{0}\right) \sin \varphi_{0} .
\end{aligned}
$$

For large indices $l$ the right hand in the above relation becomes negative. This leads us to the contradiction. Thus, there exists $m_{1} \geqslant m_{0}$ such that $\left|\gamma_{m_{1}}\left(p\left(m_{1}\right)\right)\right| \leqslant\left(a^{m_{1}} \sin \varphi_{0}\right)^{-1}$. Then by (2.26) we obtain

$$
\left|\gamma_{m_{1}+1}(0)\right|-2^{-1} \tau \sin \varphi_{0} \leqslant\left|\gamma_{m_{1}}\left(p\left(m_{1}\right)\right)\right|+\left|\gamma_{m_{1}+1}\right|-2^{-1} \tau \sin \varphi_{0} \leqslant 0 .
$$

Therefore, in view of $(2.25)$ we have $\left|\gamma_{m_{1}+1}\left(p\left(m_{1}+1\right)\right)\right| \leqslant\left(a^{m_{1}+1} \sin \varphi_{0}\right)^{-1}$. It yields that $(2.24)$ is true. It remains to show that $T$ has a zero density. In accordance with $(2.23),(2.24)$, the assumption of the lemma and the definition of $\gamma_{m}(0)$ we have:

$$
\frac{p(m)}{a^{m}} \leqslant \frac{2 a\left|\gamma_{m}(0)\right|}{\sin \varphi_{0}} \leqslant \frac{2 a\left(\left|\gamma_{m-1}(p(m-1))\right|+\left|\gamma_{m}\right|\right)}{\sin \varphi_{0}} \rightarrow 0, \quad m \rightarrow \infty .
$$

We fix $\varepsilon>0$. Then there exists an index $m(\varepsilon)$ such that $p(m) \leqslant \varepsilon a^{m}, m \geqslant m(\varepsilon)$. Let $r>a^{m(\varepsilon)}$ and an index $m(r)$ is chosen so that $a^{m(r)} \leqslant r<a^{m(r)+1}$. Then

$$
\begin{aligned}
\frac{n(r, T)}{r} & =\frac{n\left(a^{m(\varepsilon)}, T\right)}{r}+\frac{n\left(a^{m(r)+1}, T\right)-n\left(a^{m(\varepsilon)}, T\right)}{r} \\
& \leqslant \frac{n\left(a^{m(\varepsilon)}, T\right)}{r}+\frac{p(m(\varepsilon))+\cdots+p(m(r))}{a^{m(r)}} \leqslant \frac{n\left(a^{m(\varepsilon)}, T\right)}{r}+\varepsilon \frac{a^{m(\varepsilon)}+\cdots+a^{m(r)}}{a^{m(r)}} .
\end{aligned}
$$

It follows that $\bar{n}(T) \leqslant \varepsilon a /(a-1)$. Since $\varepsilon>0$ is arbitrary, this completes the proof.

Lemma 2.6. Let $\Lambda=\left\{\lambda_{k}\right\}_{k=1}^{\infty}$ has a density $\tau \geqslant 0, a>1, r_{2}>r_{1}>0$ and $r_{2} / r_{1} \leqslant a$. Then the representation

$$
\sum_{r_{1} \leqslant\left|\lambda_{k}\right|<r_{2}} \frac{1}{\left|\lambda_{k}\right|}=\tau \ln \left(\frac{r_{2}}{r_{1}}\right)+\varepsilon\left(r_{1}, r_{2}\right), \quad \varepsilon\left(r_{1}, r_{2}\right) \rightarrow 0, \quad r_{1} \rightarrow \infty, \quad r_{2}>r_{1}>0, \quad r_{2} / r_{1} \leqslant a,
$$

holds true, that is $\varepsilon\left(r_{1}, r_{2}\right) \rightarrow 0, r_{1} \rightarrow \infty$, uniformly in $r_{2}: r_{2}>r_{1}>0, r_{2} / r_{1} \leqslant a$.

Remark. If the ring $r_{1} \leqslant|\lambda|<r_{2}$ contains no points $\lambda_{k}$, we assume that the left hand side in the above identity vanishes.

Proof. We assume that $n(r, \Lambda) \rightarrow+\infty, r \rightarrow \infty$; otherwise the statement of the lemma becomes trivial.

Let $\tau=0$. Since $r_{2} / r_{1} \leqslant a$, then

$$
\sum_{r_{1} \leqslant\left|\lambda_{k}\right|<r_{2}} \frac{1}{\left|\lambda_{k}\right|} \leqslant \frac{1}{r_{1}}\left(n\left(a r_{1}, \Lambda\right)-n\left(r_{1}, \Lambda\right)\right) \rightarrow 0, \quad r_{1} \rightarrow \infty .
$$

Let $\tau>0$. Then by Euler representation we have

$$
\sum_{k=1}^{n} \frac{1}{k}=\ln n+\beta+\beta(n), \quad \beta(n) \rightarrow 0, \quad n \rightarrow \infty,
$$


where $\beta$ is the Euler constant. By the assumption, $\Lambda$ has the density $\tau$, that is, the identities

$$
\left|\lambda_{k}\right|=k /(\tau+\delta(k)), k \rightarrow \infty, \quad n(r, \Lambda)=\tau r+\varepsilon(r) r, \varepsilon(r) \rightarrow 0, r \rightarrow \infty,
$$

hold true. By (2.27) it follows that

$$
\begin{aligned}
\sum_{r_{1} \leqslant\left|\lambda_{k}\right|<r_{2}} \frac{1}{\left|\lambda_{k}\right|} & =\sum_{r_{1} \leqslant\left|\lambda_{k}\right|<r_{2}} \frac{\tau+\delta(k)}{k}=\tau \sum_{k=n\left(r_{1}, \Lambda\right)+1}^{n\left(r_{2}, \Lambda\right)} \frac{1}{k}+\sum_{k=n\left(r_{1}, \Lambda\right)+1}^{n\left(r_{2}, \Lambda\right)} \frac{\delta(k)}{k} \\
& =\tau \ln \frac{n\left(r_{2}, \Lambda\right)}{n\left(r_{1}, \Lambda\right)}+\tau\left(\beta\left(n\left(r_{2}, \Lambda\right)\right)-\beta\left(n\left(r_{1}, \Lambda\right)\right)\right)+\sum_{k=n\left(r_{1}, \Lambda\right)+1}^{n\left(r_{2}, \Lambda\right)} \frac{\delta(k)}{k} \\
& =\tau \ln \frac{r_{2}}{r_{1}}+\tau\left(\ln \frac{\tau+\varepsilon\left(r_{2}\right)}{\tau+\varepsilon\left(r_{1}\right)}+\beta\left(n\left(r_{2}, \Lambda\right)\right)-\beta\left(n\left(r_{1}, \Lambda\right)\right)\right)+\sum_{k=n\left(r_{1}, \Lambda\right)+1}^{n\left(r_{2}, \Lambda\right)} \frac{\delta(k)}{k} .
\end{aligned}
$$

We fix $\varepsilon>0$. In accordance with (2.27) and (2.28) we choose an index $k_{0}$ such that $|\delta(k)| \leqslant \varepsilon$, $\beta(n) \mid \leqslant \varepsilon, k, n \geqslant k_{0}$. By (2.28) we also choose $r(\varepsilon)>0$ such that

$$
\left|\ln \frac{\tau+\varepsilon\left(r_{2}\right)}{\tau+\varepsilon\left(r_{1}\right)}\right| \leqslant \varepsilon, \quad n\left(r_{1}, \Lambda\right) \geqslant k_{0}, \quad r_{2}>r_{1}>r(\varepsilon) .
$$

Then

$$
\left|\varepsilon\left(r_{1}, r_{2}\right)\right| \leqslant 3 \varepsilon \tau+\varepsilon \sum_{k=n\left(r_{1}, \Lambda\right)+1}^{n\left(a r_{1}, \Lambda\right)} \frac{1}{k} \leqslant 3 \varepsilon \tau+\varepsilon(\ln a+3 \varepsilon), \quad r_{2}>r_{1}>r(\varepsilon), \quad r_{2} / r_{1} \leqslant a .
$$

The proof is complete.

Let $K$ be a convex compact set. It determines uniquely a function in the class $\Sigma$ by means of the arc length of its boundary $\partial K$. For each $\varphi \in \mathbb{R}$ by $L(\varphi, K)$ we denote the intersection of the support straight line

$$
l(\varphi, K)=\left\{z: \operatorname{Re}\left(z e^{-i \varphi}\right)=H(\varphi, K)\right\}, \quad H(\varphi, K)=\sup _{z \in K} \operatorname{Re}\left(z e^{-i \varphi}\right),
$$

and the boundary $\partial K$, where $H(\varphi, K)$ is the support function of the compact set $K$. The set $L(\varphi, K)$ is either a point, which we denote by $z(\varphi, K)$, or a segment. The set $\Psi(K)$ of directions $\varphi$, for which $L(\varphi, K)$ is a segment, is at most countable. Let $\varphi_{1}, \varphi_{2} \notin \Psi(K)$, $\varphi_{2}-\varphi_{1} \in(0,2 \pi)$, and $s\left(\varphi_{1}, \varphi_{2}, K\right)$ be the length of the arc $\partial K$ connecting points $z\left(\varphi_{1}, K\right)$ and $z\left(\varphi_{2}, K\right)$ and motion on this arc is made from $z\left(\varphi_{1}, K\right)$ to $z\left(\varphi_{2}, K\right)$ in the positive direction (counterclockwise). Let $\varphi_{1}, \varphi_{2} \in(-2 \pi, 0) \backslash \Psi(K), \varphi \in\left(\varphi_{1}, \varphi_{1}+2 \pi\right) \backslash \Psi(K)$. The function

$$
\omega\left(\varphi_{1}, K\right)=-\lim _{\varphi_{2} \rightarrow 0} s\left(\varphi_{1}, \varphi_{2}, K\right), \quad \omega(\varphi, K)=s\left(\varphi_{1}, \varphi, K\right)+\omega\left(\varphi_{1}, K\right)
$$

is continued uniquely to the function in the class $\Sigma$ and this continuation is independent of $\varphi_{1}$. It is easy to observe that the sets $\Psi(K) \bigcap[-2 \pi, 2 \pi)$ and $\Phi(\omega(\cdot, K))$ coincide.

Let $\varphi_{s} \notin \Phi(K), s=\overline{1, p}$, be such that $\varphi_{1} \in(-2 \pi, 0)$ and $\varphi_{1}<\cdots<\varphi_{p}<\varphi_{1}+2 \pi=\varphi_{p+1}$. We let $a_{s}=z\left(\varphi_{s}, K\right), s=\overline{1, p+1}$ and consider the convex polygon $\Omega$ with vertices $a_{1}, \ldots, a_{p}, a_{p+1}=$ $a_{1}$ inscribed in the compact set $K$. We note that some vertices with neighbouring indices can coincide. By the symbol $e_{s}$ we denote the unit outward normal to $\partial \Omega$ in the internal points (if they exist) of the segment $\left[a_{s}, a_{s+1}\right]$. We mention that for some $\varphi(s) \in\left(\varphi_{s}, \varphi_{s+1}\right)$ the identity $e_{s}=e^{i \varphi(s)}$ holds true. If $a_{s}=a_{s+1}$, then by $e_{s}$ we mean an arbitrarily chosen vector $e^{i \varphi}$, where $\varphi \in\left(\varphi_{s}, \varphi_{s+1}\right)$. The next statement has a simple geometric meaning.

Lemma 2.7. The identity

$$
\sum_{s=1}^{p}\left|a_{s+1}-a_{s}\right| e_{s}=0
$$


holds true.

Proof. We have

$$
\sum_{s=1}^{p}\left|a_{s+1}-a_{s}\right| e_{s}=\sum_{s=1}^{p}\left(a_{s+1}-a_{s}\right) e^{-i \pi / 2}=0 .
$$

Remark. It follows from 2.7 that for $\omega=\omega(\cdot, K)$, the identity

$$
\int_{0}^{2 \pi} e^{i \varphi} d \omega(\varphi)=0
$$

holds true. Vice versa, let $\omega \in \Sigma$ satisfy this identity. Then by [3, Ch. I, Sect. 17, Thm. 24], [2, Ch. I, Sect. 2, Thm. 1.2.4] the function

$$
H(\varphi)=A \cos \varphi+B \sin \varphi-\frac{1}{2 \pi} \int_{\varphi-2 \pi}^{\varphi}(\varphi-\theta) \sin (\varphi-\theta) d \omega(\theta)
$$

coincide with the support function $H(\varphi, K)$ of the convex set $K$ (for different $A, B \in \mathbb{R}$ the compact sets can be obtained one from the other by a shift). At that, $\omega(\theta) \equiv \omega(\theta, K)$.

By the symbol $\Sigma_{0}$ we denote a subclass of all functions $\omega \in \Sigma$ satisfying (2.29).

Lemma 2.8. Let $\Lambda=\left\{\lambda_{k}\right\}_{k=1}^{\infty}$ has an angular density $\omega_{\Lambda} \in \Sigma_{0}$. Then for all $a>1$, $r_{2}>r_{1}>0$ and $r_{2} / r_{1} \leqslant a$

$$
\mathcal{N}\left(r_{2}, \Lambda\right)-\mathcal{N}\left(r_{1}, \Lambda\right)=\varepsilon\left(r_{1}, r_{2}\right) \rightarrow 0, \quad r_{1} \rightarrow \infty .
$$

Proof. By the assumption, $\omega_{\Lambda} \in \Sigma_{0}$. Then, as it was mentioned above, there exists a convex compact set $K$ obeying the identity $\omega_{\Lambda} \equiv \omega(\varphi, K)$.

We fix $\varepsilon>0$ and choose $\delta>0$ such that

$$
\left|e^{i \varphi}-e^{i \theta}\right| \leqslant \varepsilon /(4 s(K) \ln a), \quad \forall \varphi, \theta:|\varphi-\theta|<\delta,
$$

where $s(K)=\omega_{\Lambda}\left(\varphi_{1}+2 \pi\right)-\omega_{\Lambda}\left(\varphi_{1}\right)$ is the length of the boundary of the compact set $K$. We choose numbers $\varphi_{s} \notin \Phi\left(\omega_{\Lambda}\right), s=\overline{1, p}, \varphi_{1} \in(-2 \pi, 0), \varphi_{1}<\cdots<\varphi_{p}<\varphi_{1}+2 \pi=\varphi_{p+1}$, satisfying the conditions: 1) $\left.\varphi_{s+1}-\varphi_{s}<\delta, s=\overline{1, p}, 2\right) s(K)-P(\Omega)<\varepsilon /(4 \ln a)$, where $P(\Omega)$ is the perimeter of the convex polygon $\Omega$ with the vertices $a_{1}, \ldots, a_{p}, a_{p+1}=a_{1}, a_{s}=z\left(\varphi_{s}, K\right)$, $s=\overline{1, p+1}$.

Let $\lambda_{k}=\left|\lambda_{k}\right| e^{i \psi_{k}}, \psi_{k} \in\left(\varphi_{1}, \varphi_{1}+2 \pi\right], k \geqslant 1$, and $\varphi(s) \in\left(\varphi_{s}, \varphi_{s+1}\right), s=\overline{1, p}$, are such that the vector $e_{s}=e^{i \varphi(s)}$ is the outward normal to $\partial \Omega$ in the internal points of the segment $\left[a_{s}, a_{s+1}\right]$ (if there are no such points, then $\varphi(s) \in\left(\varphi_{s}, \varphi_{s+1}\right)$ is chosen arbitrarily). Then by condition 1) and the choice of $\delta>0$ we have:

$$
\left|\sum_{\psi_{k} \in\left(\varphi_{s}, \varphi_{s+1}\right], r_{1} \leqslant\left|\lambda_{k}\right|<r_{2}}\left(\frac{1}{\lambda_{k}}-\frac{1}{\left|\lambda_{k}\right| e^{i \varphi(s)}}\right)\right| \leqslant \frac{\varepsilon}{4 s(K) \ln a} \sum_{\psi_{k} \in\left(\varphi_{s}, \varphi_{s+1}\right], r_{1} \leqslant\left|\lambda_{k}\right|<r_{2}} \frac{1}{\left|\lambda_{k}\right|} .
$$

Since $n\left(\Lambda\left(\varphi_{s}, \varphi_{s+1}\right]\right)=\omega_{\Lambda}\left(\varphi_{s+1}\right)-\omega_{\Lambda}\left(\varphi_{s}\right)$, by Lemma 2.6 we obtain

$$
\left|\sum_{\psi_{k} \in\left(\varphi_{s}, \varphi_{s+1}\right], r_{1} \leqslant\left|\lambda_{k}\right|<r_{2}}\left(\frac{1}{\lambda_{k}}-\frac{1}{\left|\lambda_{k}\right| e^{i \varphi(s)}}\right)\right| \leqslant \frac{\epsilon\left(\omega_{\Lambda}\left(\varphi_{s+1}\right)-\omega_{\Lambda}\left(\varphi_{s}\right)\right)}{4 s(K)}+\varepsilon_{s}\left(r_{1}, r_{2}\right),
$$

where $r_{2}>r_{1}>0, r_{2} / r_{1} \leqslant a$ and $\varepsilon_{s}\left(r_{1}, r_{2}\right) \rightarrow 0, r_{1} \rightarrow \infty$. Thus,

$$
\sum_{s=1}^{p}\left|\sum_{\psi_{k} \in\left(\varphi_{s}, \varphi_{s+1}\right], r_{1} \leqslant\left|\lambda_{k}\right|<r_{2}}\left(\frac{1}{\lambda_{k}}-\frac{1}{\left|\lambda_{k}\right| e^{i \varphi(s)}}\right)\right| \leqslant \frac{\varepsilon}{4}+\widetilde{\varepsilon}\left(r_{1}, r_{2}\right) \leqslant \frac{\varepsilon}{2},
$$


where $r_{2}>r_{1}>r_{0}(\varepsilon), \frac{r_{2}}{r_{1}} \leqslant a$.

Let $\omega_{\Lambda}\left(\varphi_{s+1}\right)-\omega_{\Lambda}\left(\varphi_{s}\right)=\left|a_{s+1}-a_{s}\right|+\gamma_{s}$. Employing again Lemma 2.6 and Lemma 2.7, we get

$$
\begin{aligned}
\sum_{s=1}^{p} \sum_{\psi_{k} \in\left(\varphi_{s}, \varphi_{s+1}\right], r_{1} \leqslant\left|\lambda_{k}\right|<r_{2}} \frac{\bar{e}_{s}}{\left|\lambda_{k}\right|} & =\ln \left(\frac{r_{2}}{r_{1}}\right) \sum_{s=1}^{p} \bar{e}_{s}\left(\left|a_{s+1}-a_{s}\right|+\gamma_{s}\right)+\hat{\varepsilon}\left(r_{1}, r_{2}\right) \\
& =\ln \left(\frac{r_{2}}{r_{1}}\right) \sum_{s=1}^{p} \bar{e}_{s} \gamma_{s}+\hat{\varepsilon}\left(r_{1}, r_{2}\right), \quad \hat{\varepsilon}\left(r_{1}, r_{2}\right) \rightarrow \rightarrow 0, \quad r_{1} \rightarrow \infty .
\end{aligned}
$$

In view of (2.30) and condition 2) we obtain

$$
\begin{aligned}
& \mathcal{N}\left(r_{2}, \Lambda\right)-\mathcal{N}\left(r_{1}, \Lambda\right)=\varepsilon\left(r_{1}, r_{2}\right), \\
& \left|\varepsilon\left(r_{1}, r_{2}\right)\right| \leqslant \frac{\varepsilon}{2}+\ln \left(\frac{r_{2}}{r_{1}}\right)\left|\sum_{s=1}^{p} \bar{e}_{s} \gamma_{s}\right|+\left|\hat{\varepsilon}\left(r_{1}, r_{2}\right)\right| \leqslant \frac{3 \varepsilon}{4}+\ln a(s(K)-P(\Omega)) \leqslant \varepsilon,
\end{aligned}
$$

where $r_{2}>r_{1}>\widetilde{r}_{0}(\varepsilon), r_{2} / r_{1} \leqslant a$. This completes the proof.

Let $\omega \in \Sigma$. we shall say that $\omega$ is a general form function if there exist $\varphi_{1}, \varphi_{2}, \varphi_{3} \in[-\pi, \pi)$ such that $\varphi_{1}<\varphi_{2}<\varphi_{3}, \varphi_{2}-\varphi_{1}<\pi, \varphi_{3}-\varphi_{2}<\pi, \varphi_{1}+2 \pi-\varphi_{3}<\pi$ and

$$
\omega\left(\varphi_{j}+\varphi\right)-\omega\left(\varphi_{j}-\varphi\right)>0, \quad j=1,2,3, \quad \varphi \in(0, \pi / 2) .
$$

If $\Lambda$ has an angular density $\omega$, it is easy to confirm that $\Lambda$ is a general form sequence if and only if $\omega=\omega_{\Lambda}$ is a general form function. Let $K$ be a convex compact set. It is easy to show that $\omega(\varphi, K)$ is a general form function if and only if it is a closure of a bounded convex domain. Assume that the identity $\omega(\varphi) \equiv \omega(\varphi, K)$ holds true. If $K$ is a point, then $\omega(\varphi) \equiv 0$. If $K$ is a segment, then $\omega$ takes exactly three mutually different values in $[0,2 \pi]$. In other cases, that is, as $K$ is a closure of a domain, $\omega$ takes more than three mutually different values in segment $[0,2 \pi]$. If $\omega \in \Sigma_{0}$, as it was mentioned above, there exists a convex compact set $K$ satisfying the identity $\omega(\varphi) \equiv \omega(\varphi, K)$.

Thus, if $\omega \in \Sigma_{0}$, then $\omega$ is a general form function if and only if it takes more than three mutually different values in segment $[0,2 \pi]$.

Lemma 2.9. Let $\widetilde{\Lambda}$ has an angular density $\omega_{\widetilde{\Lambda}} \in \Sigma_{0}$ and $\omega_{\widetilde{\Lambda}}$ is a general form function. Then there exists a sequence $\Lambda \subseteq \widetilde{\Lambda}$ with an angular density $\omega_{\Lambda}=\omega_{\widetilde{\Lambda}}$ such that $\mathcal{N}(r, \Lambda) \rightarrow 0$, $r \rightarrow+\infty$.

Proof. We let

$$
\gamma_{1}=\mathcal{N}\left(2^{2}, \widetilde{\Lambda}\right), \quad \gamma_{m}=\mathcal{N}\left(2^{m+1}, \widetilde{\Lambda}\right)-\mathcal{N}\left(2^{m}, \widetilde{\Lambda}\right), \quad m \geqslant 2 .
$$

Since $\omega_{\tilde{\Lambda}} \in \Sigma_{0}$, by Lemma $2.8 \gamma_{m} \rightarrow 0, m \rightarrow \infty$. By the assumption, $\omega_{\tilde{\Lambda}}$ is a general form function. Therefore, $\widetilde{\Lambda}$ is a general form sequence. Then by Lemma 2.5 there exists a sequence of zero density $T \subset \widetilde{\Lambda}$ such that

$$
\sum_{m=1}^{l} \gamma_{m}-\mathcal{N}\left(2^{l+1}, T\right) \rightarrow 0, \quad l \rightarrow \infty .
$$

By the definition of $\gamma_{m}$ we obtain: $\mathcal{N}\left(2^{l}, \widetilde{\Lambda}\right)-\mathcal{N}\left(2^{l}, T\right) \rightarrow 0, l \rightarrow \infty$. Let $\Lambda \subseteq \widetilde{\Lambda}$ is a sequence completing $T$ to $\widetilde{\Lambda}$, that is, $\widetilde{\Lambda}=\Lambda \bigcup T$. Then by the above facts we have $\mathcal{N}\left(2^{l}, \Lambda\right) \rightarrow 0$, $l \rightarrow+\infty$. For each $r>0$ we choose a number $l(r)$ by condition $2^{l(r)} \leqslant r<2^{l(r)+1}$. The proven facts and Lemma 2.8 yield

$$
N(r, \Lambda)=N\left(2^{l(r)}, \Lambda\right)+\left(\mathcal{N}(r, \Lambda)-\mathcal{N}\left(2^{l(r)}, \Lambda\right)\right) \rightarrow 0, \quad r \rightarrow+\infty .
$$


Since $T$ has a zero density, the sequence $\Lambda$ has an angular density $\omega_{\Lambda}=\omega_{\widetilde{\Lambda}}$. At that, the embedding holds true. The proof is complete.

Theorem 2. Let $\delta>0$ and $\omega \in \Sigma_{0}$ is a general form function. Then there exists a sequence $\Lambda=\left\{\lambda_{k}\right\}_{k=1}^{\infty} \subseteq \Lambda_{\mathbb{Z}}$ with an angular density $\omega_{\Lambda}=\omega$ such that

$$
\left|\lambda_{k+1}\right|-\left|\lambda_{k}\right| \geqslant \alpha=\frac{1}{2\left(\omega_{\Lambda}\left(\varphi_{1}+2 \pi\right)-\omega_{\Lambda}\left(\varphi_{1}\right)\right)}-\delta, \quad k \geqslant 1,
$$

where $\varphi_{1} \in(-2 \pi, 0) \backslash \Phi(\omega)$ is chosen arbitrarily and $\mathcal{N}(r, \Lambda) \rightarrow 0, r \rightarrow+\infty$.

Proof. By Theorem 2.1, there exists a sequence $\widetilde{\Lambda}=\left\{\widetilde{\lambda}_{k}\right\}_{k=1}^{\infty} \subseteq \Lambda_{\mathbb{Z}}$ with an angular density $\omega_{\tilde{\Lambda}}=\omega$ such that

$$
\left|\widetilde{\lambda}_{k+1}\right|-\left|\widetilde{\lambda}_{k}\right| \geqslant \alpha, \quad k \geqslant 1
$$

By Lemma 2.9, there exists a sequence $\Lambda \subseteq \widetilde{\Lambda} \subseteq \Lambda_{\mathbb{Z}}$ with an angular density $\omega_{\Lambda}=\omega_{\widetilde{\Lambda}}=\omega$ satisfying the condition $\mathcal{N}(r, \Lambda) \rightarrow 0, r \rightarrow+\infty$. It remains to observe that inequalities (2.31) hold true for $\Lambda \subseteq \widetilde{\Lambda}$ by (2.32). The proof is complete.

Remark. The sequence $\Lambda \subseteq \Lambda_{\mathbb{Z}}$, existence of which is proved in Theorem 2.2, is a regular set and in particular, it is properly distributed.

\section{REPRESENTATION OF ANALYTIC FUNCTIONS}

Properly distributed sets are closely related with functions of regular growth. Let $f$ be an entire function of exponential type, that is, there exists $A>0$ and $B>0$ such that

$$
\ln |f(\lambda)| \leqslant A+B|\lambda|, \quad \lambda \in \mathbb{C} .
$$

The upper indicator of $f$ (or simply indicator) is the function

$$
h_{f}(\lambda)=\varlimsup_{t \rightarrow \infty} \frac{\ln |f(t \lambda)|}{t}, \quad \lambda \in \mathbb{C} .
$$

The indicator $h_{f}$ is a convex positive homogeneous function of order one. At that, $h_{f}\left(e^{i \varphi}\right)$ coincides with the support function $H(\varphi, K)$ of some convex compact set $K$ called the indicator diagram of $f$ (see, [3, Ch. I, Sect. 19]). The compact set complex conjugated with $K$ is called the conjugate diagram of function $f$.

Function $f$ is said to have a regular growth (see $[3$, Ch. III]) if

$$
h_{f}(\lambda)=\lim _{t \rightarrow \infty, t \notin E} \frac{\ln |f(t \lambda)|}{t}, \quad \lambda \in \mathbb{C},
$$

where $E$ is a set of zero relative measure in the ray $(0,+\infty)$, that is, the Lebesgue measure of its intersection with the interval $(0, r)$ is infinitesimally small in comparison with $r$ as $r \rightarrow+\infty$. The regularity of the growth for function $f$ is equivalent to the asymptotic identity

$$
\ln |f(\lambda)|=h_{f}(\lambda)+\alpha(\lambda), \quad \lambda \in \mathbb{C}, \quad \lim _{|\lambda| \rightarrow \infty, \lambda \notin \mathcal{I}_{f}} \alpha(\lambda) /|\lambda|=0,
$$

where $\mathcal{I}_{f}$ is a some $C^{0}$-set. We recall (see [3, Ch. II, Sect. 1]) that $\mathcal{R} \subset \mathbb{C}$ is called $C^{0}$-set if it can be covered by the balls $B\left(z_{j}, r_{j}\right), j \geqslant 1$, such that

$$
\lim _{r \rightarrow \infty} \frac{1}{r} \sum_{\left|z_{j}\right|<r} r_{j}=0 .
$$

Let $\Lambda=\left\{\lambda_{k}\right\}_{k=1}^{\infty}$ and $f(\lambda, \Lambda)$ be the canonical product

$$
f(\lambda, \Lambda)=\prod_{k=1}^{\infty}\left(1-\frac{\lambda}{\lambda_{k}}\right) \exp \frac{n_{k} \lambda}{\lambda_{k}} .
$$


The function $f(\lambda, \Lambda)$ has a regular growth if and only if $\Lambda$ is a properly distributed set [3, Ch. III, Sect. 3, Thm. 4, Ch. II, Sect. 1, Thm. 2]. At that, its angular density $\omega_{\Lambda}$ belongs to the set $\Sigma_{0}$. If $K$ is an indicator diagram of the function $f(\lambda, \Lambda)$, then $\omega_{\Lambda}(\theta) \equiv \omega(\theta, K) / 2 \pi$ and $h_{f}\left(e^{i \varphi}\right) \equiv H(\varphi, K)[3$, Ch. II, Sect. 1, Eq. (2.07)].

Let $D$ be a bounded convex domain in $\mathbb{C}$ and $H(\bar{D})$ is a space of functions analytic in the vicinity of the closure $\bar{D}$. There are well-known A.F. Leontiev conditions $[2, \mathrm{Ch}$. IV, Sect. 6, Thm. 4.6.4] for representing functions $g \in H(\bar{D})$ as the series

$$
g(z)=\sum_{k=1}^{\infty} d_{k} e^{\lambda_{k} z}, \quad z \in D
$$

in the case, when $\Lambda$ is a set of simple zeroes for an entire function $f$ of exponential type, whose conjugated diagram coincides with $\bar{D}$. These are two conditions: the regularity of the growth of function $f$ and the lower bound for the absolute value of its derivatives at points $\lambda_{k}$

$$
\ln \left|f^{\prime}\left(\lambda_{k}\right)\right| \geqslant h_{f}\left(\lambda_{k}\right)-\varepsilon_{k}\left|\lambda_{k}\right|, \quad 0<\varepsilon_{k} \rightarrow 0, \quad k \rightarrow \infty .
$$

Let us provide sufficient conditions for representation (3.1) for an arbitrary sequence $\Lambda=$ $\left\{\lambda_{k}\right\}_{k=1}^{\infty}$, which sequence is not assumed to be a zero set of some entire functions. These conditions are formulated only in terms of geometric characteristics of $\Lambda$ and $D$. In order to do it, we need a "local" characteristics of the sequence $\Lambda$ introduced in work [7].

Let $\Lambda=\left\{\lambda_{k}\right\}_{k=1}^{\infty}$. We consider the function

$$
q_{\Lambda}(z, w, \delta)=\prod_{\lambda_{k} \in B(w, \delta|w|)} \frac{z-\lambda_{k}}{3 \delta\left|\lambda_{k}\right|}
$$

In the case, when the ball $B(w, \delta|w|)$ contains no points $\lambda_{k}$, we let $q_{\Lambda}(z, w, \delta) \equiv 1$. The absolute value of the function $q_{\Lambda}(z, w, \delta)$ can be interpreted as the measure of accumulation of points $\lambda_{k} \in B(w, \delta|w|)$ at $z$. The quantity $\ln \left|q_{\Lambda}(z, w, \delta)\right| /|w|$ is similar by sense to the logarithm of geometric mean (arithmetic mean of logarithm) of normalized distances from $\lambda_{k} \in B(w, \delta|w|)$ to $z$. If $\delta \in(0,1)$, then the absolute value of each factor in the definition of $q_{\Lambda}$ in the ball $B(w, \delta|w|)$ is estimated from above by the quantity $2(3(1-\delta))^{-1}$. This is why for $\delta \in(0,1 / 3)$ it does not exceed one. We let

$$
q_{\Lambda}^{m}(z, \delta)=\prod_{\lambda_{k} \in B\left(\lambda_{m}, \delta\left|\lambda_{m}\right|\right), k \neq m} \frac{z-\lambda_{k}}{3 \delta\left|\lambda_{k}\right|}, \quad S_{\Lambda}=\lim _{\delta \rightarrow 0} \varlimsup_{m \rightarrow \infty} \frac{\ln \left|q_{\Lambda}^{m}\left(\lambda_{m}, \delta\right)\right|}{\left|\lambda_{m}\right|} .
$$

The definition of $S_{\Lambda}$ implies the inequality $S_{\Lambda} \leqslant 0$ (see [7]).

Lemma 3.1. Let $\Lambda=\left\{\lambda_{k}\right\}_{k=1}^{\infty}$ be the zero set of an entire function $f$ of exponential type and regular growth. Assume that $S_{\Lambda}=0$. Then

$$
\ln \left|f^{\prime}\left(\lambda_{k}\right)\right| \geqslant h_{f}\left(\lambda_{k}\right)-\varepsilon_{k}\left|\lambda_{k}\right|, \quad 0<\varepsilon_{k} \rightarrow 0, \quad k \rightarrow \infty .
$$

Proof. The regular growth of function $f$ means that

$$
\ln |f(\lambda)|=h_{f}(\lambda)+\alpha(\lambda), \quad \lambda \in \mathbb{C}, \quad \lim _{|\lambda| \rightarrow \infty, \lambda \notin \mathcal{I}_{f}} \alpha(\lambda) /|\lambda|=0,
$$

where $\mathcal{I}_{f}$ is a $C^{0}$-set. Fix $\varepsilon>0$ and choose $R>0$ such that

$$
\alpha(\lambda) \geqslant-\varepsilon|\lambda|, \quad \lambda \in \mathcal{I}_{f}, \quad|\lambda| \geqslant R
$$

The indicator $h_{f}(\lambda)$ is a convex function and this is why it is continuous. Employing its uniform continuity on compact subsets, we find $\delta_{0} \in(0,1 / 3)$, for which the inequality

$$
\left|h_{f}(\lambda)-h_{f}(w)\right| \leqslant \varepsilon, \quad w \in B\left(\lambda, \delta_{0}\right), \quad|\lambda|=1,
$$


holds true. In accordance with the assumption and the definition of quantity $S_{\Lambda}$ we choose $\delta \in\left(0, \delta_{0}\right)$ and an index $k_{0}$ such that

$$
\left|\lambda_{k}\right| \geqslant 2 R, \quad \ln \left|q_{\Lambda}^{k}\left(\lambda_{k}, \delta\right)\right| \geqslant-\varepsilon\left|\lambda_{k}\right|, \quad k \geqslant k_{0} .
$$

Finally, taking into consideration that $\mathcal{I}_{f}$ is a $C^{0}$-set, we can assume the following condition: for each $k \geqslant k_{0}$ the total sum of exceptional circles in $\mathcal{I}_{f}$ intersecting $B\left(\lambda_{k}, \delta\left|\lambda_{k}\right|\right)$ does not exceed $\delta\left|\lambda_{k}\right| / 4$. Then by (3.3), (3.4) and (3.6), for each $k \geqslant k_{0}$, there exists $\alpha_{k} \in(1 / 2,1)$ such that

$$
\ln |f(\lambda)| \geqslant h_{f}(\lambda)-\varepsilon|\lambda|, \quad \lambda \in \partial B\left(\lambda_{k}, \alpha_{k} \delta\left|\lambda_{k}\right|\right)
$$

Taking into consideration the positive homogeneity of the indicator and (3.5), we obtain:

$$
\ln |f(\lambda)| \geqslant h_{f}\left(\lambda_{k}\right)-3 \varepsilon\left|\lambda_{k}\right|, \quad \lambda \in \partial B\left(\lambda_{k}, \alpha_{k} \delta\left|\lambda_{k}\right|\right), \quad k \geqslant k_{0} .
$$

According to the assumption, the function $\ln \mid\left(f(\lambda) / q_{\Lambda}\left(\lambda, \lambda_{k}, \delta\right) \mid\right.$ is harmonic in the circle $B\left(\lambda_{k}, \delta\left|\lambda_{k}\right|\right)$. Since $\delta<1 / 3$, the function $\ln \left|q_{\Lambda}\left(\lambda, \lambda_{k}, \delta\right)\right|$ is non-positive in this circle. Hence, employing (3.7) and the minimum principle for harmonic functions, we get:

$$
\ln \left|h_{k}\left(\lambda_{k}\right)\right| \geqslant h_{f}\left(\lambda_{k}\right)-3 \varepsilon\left|\lambda_{k}\right|, \quad k \geqslant k_{0} .
$$

By (3.6) this implies:

$$
\ln \left|f^{\prime}\left(\lambda_{k}\right)\right|=\ln \left|h_{k}\left(\lambda_{k}\right)\right|+\ln \left|q_{\Lambda}^{k}\left(\lambda_{k}, \delta\right)\right|-\ln \left(3 \delta\left|\lambda_{k}\right|\right) \geqslant h_{f}\left(\lambda_{k}\right)-5 \varepsilon\left|\lambda_{k}\right|, \quad k \geqslant k_{1} .
$$

The proof is complete.

Remark. 1. Estimate (3.2) implies the identity $S_{\Lambda}=0$. At that, the regularity of the growth of the function $f$ is not needed (see the proof of Corollary 4.2 in work [87).

2. The issue on whether estimate (3.2) implies the regularity of the growth for $f$ is still open. The answer is the matter of the A.F. Leontiev problem.

3. The only identity $S_{\Lambda}=0$ does not imply estimate (3.2) (see example in the end of work $[9])$.

Theorem 3. Let $D$ be a bounded convex domain and $\widetilde{\Lambda}=\left\{\widetilde{\lambda}_{k}\right\}_{k=1}^{\infty}$ has an angular density. Assume that $S_{\widetilde{\Lambda}}=0$ and the identity $\omega_{\widetilde{\Lambda}}(\varphi) \equiv \omega(\varphi, \widetilde{K}) / 2 \pi$ holds true, where $\widetilde{K}$ is the complex conjugate to $\bar{D}$ compact set. Then in the domain $D$ each function $g \in H(\bar{D})$ is represented by the series

$$
g(z)=\sum_{k=1}^{\infty} \widetilde{d}_{k} e^{\tilde{\lambda}_{k} z}, \quad z \in D .
$$

Proof. Since $D$ is a domain, then $\omega(\psi, \widetilde{K}) \in \Sigma_{0}$ (see the remark after Lemma 2.7 ) and $\omega(\psi, \widetilde{K})$ is a general form function. Then in accordance with Lemma 2.9, there exists a properly distributed set $\Lambda \subseteq \widetilde{\Lambda}$ with an angular density $\omega_{\Lambda}=(2 \pi)^{-1} \omega(\cdot, \widetilde{K})$. In the beginning of the section we mentioned that in this case the canonical function $f(\lambda, \Lambda)$ has a regular growth, while its indicator diagram (see the remark after Lemma 2.7) coincides with some shift $\widetilde{K}-z_{0}$ of the compact set $\widetilde{K}$.

We let $f(\lambda)=f(\lambda, \Lambda) e^{\lambda \bar{z}_{0}}$. Then the function $f$ has a regular growth and its conjugate diagram coincides with $\bar{D}$. By the assumption, $S_{\Lambda}=0$. Since $\Lambda \subseteq \widetilde{\Lambda}$, by the definition all the factors forming the function $q_{\Lambda}^{m}(z, \delta)$ are among the factors forming the function $q_{\widetilde{\Lambda}}^{m}(z, \delta)$. As $\delta \in(0,1 / 3)$, the absolute value of each of them does not exceed one. It implies the inequality $S_{\Lambda} \geqslant S_{\widetilde{\Lambda}}=0$. We mentioned above that we always have $S_{\Lambda}=0$. This is why $S_{\Lambda}=0$.

Thus, we can apply Lemma 3.1. In accordance with this lemma, estimate (3.2) holds true. Therefore, by Theorem 4.6.4. in book [2], each function $g \in H(\bar{D})$ is represented in the domain $D$ by series (3.1), and therefore, by series (3.8). The proof is complete. 
Remark. Let $\left\{K_{p}\right\}_{p=1}^{\infty}$ be a sequence of convex compact sets in the domain D, which strictly exhausts it, that is, $K_{p} \subset$ int $K_{p+1}, p \geqslant 1$, (the symbol int stands for the interior of a set) and $D=\bigcup_{p=1}^{\infty} K_{p}$. For each $p \geqslant 1$ we introduce the Banach space of the sequences of complex numbers

$$
Q_{p}=\left\{d=\left\{d_{k}\right\}:\|d\|_{p}=\sup _{k \geqslant 1}\left|d_{k}\right| \exp H_{K_{p}}\left(\lambda_{k}\right)<\infty\right\}
$$

Let $Q(D, \Lambda)=\bigcap_{p \geqslant 1} Q_{p}$ be equipped with the topology of the projective limit. According to Lemma 2.3 in work [1], the pointwise convergence of series (3.1) on the domain D implies the inclusion $d=\left\{d_{k}\right\} \in Q(D, \Lambda)$. Moreover, by Theorem 3.1 in this work (an analogue of Abel theorem for power series) the inequality

$$
\sum_{k=1}^{\infty}\left|d_{k}\right| \max _{z \in K_{p}}\left|e^{\lambda_{k} z}\right| \leqslant\left. C_{p}|| d\right|_{p+2}, \quad p \geqslant 1,
$$

holds true, where $C_{p}>0$ is independent of $d=\left\{d_{k}\right\} \in Q_{p}$. In particular, it means that series (3.1) ((3.8)) converges absolutely and uniformly on each compact set on the domain D.

Lemma 3.2. Let $\alpha>0$ and $\Lambda=\left\{\lambda_{k}\right\}_{k=1}^{\infty}$ satisfy the condition

$$
\left|\lambda_{k+1}\right|-\left|\lambda_{k}\right| \geqslant \alpha, \quad k \geqslant 1
$$

Then $S_{\Lambda}=0$.

Proof. Let $m \geqslant 1$ and $\delta \in(0,1 / 3)$. In view of (3.9), for each $\lambda_{k} \in B\left(\lambda_{m}, \delta\left|\lambda_{m}\right|\right)$ we have:

$$
\delta\left|\lambda_{m}\right|>\left|\lambda_{m}-\lambda_{k}\right| \geqslant|| \lambda_{m}|-| \lambda_{k}|| \geqslant|m-k| \alpha .
$$

Therefore, the inequalities $(1-\delta)\left|\lambda_{m}\right|<\left|\lambda_{k}\right|<(1+\delta)\left|\lambda_{m}\right|$ hold true. By $l(m, \delta)$ we denote the maximal natural number obeying $l(m, \delta) \alpha<\delta\left|\lambda_{m}\right|$. Then the quantity $l \alpha / 3 \delta\left|\lambda_{k}\right|$ does not exceed one for all $l=\overline{1, l(m, \delta)}$. This is why the above facts yield (we also take into consideration that $\left.s ! \geqslant(s / 3)^{s}\right)$

$$
\begin{aligned}
\left|q_{\Lambda}^{m}\left(\lambda_{m}, \delta\right)\right| & \geqslant \prod_{\lambda_{k} \in B\left(\lambda_{m}, \delta\left|\lambda_{m}\right|\right), k \neq m} \frac{\left|\lambda_{m}-\lambda_{k}\right|}{3 \delta\left|\lambda_{k}\right|} \geqslant \prod_{\lambda_{k} \in B\left(\lambda_{m}, \delta\left|\lambda_{m}\right|\right), k \neq m} \frac{|m-k| \alpha}{3 \delta\left|\lambda_{k}\right|} \\
& \geqslant \prod_{l=1}^{l(m, \delta)}\left(\frac{l \alpha}{3 \delta\left|\lambda_{k}\right|}\right)^{2}=\left(\frac{\alpha}{(1+\delta) 3 \delta\left|\lambda_{m}\right|}\right)^{2 l(m, \delta)}(l(m, \delta) !)^{2} \geqslant\left(\frac{l(m, \delta) \alpha}{(1+\delta) 9 \delta\left|\lambda_{m}\right|}\right)^{2 l(m, \delta)} .
\end{aligned}
$$

Thus, by the definition of $l(m, \delta)$ we have

$$
\begin{aligned}
& S_{\Lambda} \geqslant \lim _{\delta \rightarrow 0} \frac{\lim }{m \rightarrow \infty} \frac{2 l(m, \delta)}{\left|\lambda_{m}\right|} \ln \frac{l(m, \delta) \alpha}{(1+\delta) 9 \delta\left|\lambda_{m}\right|} \geqslant \lim _{\delta \rightarrow 0} \underline{\lim _{m \rightarrow \infty}} \frac{2 l(m, \delta)}{\left|\lambda_{m}\right|} \ln \frac{\delta\left|\lambda_{m}\right|-\alpha}{(1+\delta)=9 \delta\left|\lambda_{m}\right|} \\
& =\lim _{\delta \rightarrow 0} \frac{\lim }{m \rightarrow \infty} \frac{2 l(m, \delta)}{\left|\lambda_{m}\right|} \ln \frac{1}{(1+\delta) 9} \geqslant \lim _{\delta \rightarrow 0} \frac{\lim }{m \rightarrow \infty} \frac{2 \delta\left|\lambda_{m}\right|}{\alpha\left|\lambda_{m}\right|} \ln \frac{1}{(1+\delta) 9}=0 .
\end{aligned}
$$

Since we always have $S_{\Lambda} \leqslant 0$, this completes the proof.

Theorem 4. Let $D$ be a bounded convex domain in $\mathbb{C}$. Then each function $g \in H(\bar{D})$ is represented by the series

$$
g(z)=\sum_{m, l \in \mathbb{Z}} d_{m, l} e^{(m+i l) z}, \quad z \in D .
$$

At that, $\left\{d_{m, l}\right\} \in Q\left(D, \Lambda_{\mathbb{Z}}\right)$ and series (3.10) converges absolutely and uniformly on compact subsets on the domain $D$. 
Proof. Let $\widetilde{K}$ be the compact set complex conjugate to $\bar{D}$. Since $D$ is a domain, then $\omega(\psi, \widetilde{K}) \in \Sigma_{0}$ (see the remark after Lemma 2.7) and $\omega(\psi, \widetilde{K})$ is a general form function. Then by Theorem 2.2 and Lemma 3.2 there exists a sequence $\Lambda=\left\{\lambda_{k}\right\}_{k=1} \subseteq \Lambda_{\mathbb{Z}}$ with the angular density $\omega_{\Lambda}=(2 \pi)^{-1} \omega(\cdot, \widetilde{K})$ such that $S_{\Lambda}=0$. By Theorem 3.1 each function $g \in H(\bar{D})$ is represented in the domain $D$ by series (3.1), and therefore, by series (3.10), where we let $d_{m, l}=d_{k}$ if $(m+i l)=\lambda_{k} \in \Lambda$ and $d_{m, l}=0$ if $(m+i l) \notin \Lambda$. At that, according to the remark after Theorem 3.1, series (3.10) converges absolutely and uniformly on compact subsets in the domain $D$. Moreover, $\left\{d_{k}\right\} \in Q(D, \Lambda)$. Together with the definition of the coefficients $d_{m, l}$ it implies that $\left\{d_{m, l}\right\} \in Q\left(D, \Lambda_{\mathbb{Z}}\right)$. The proof is complete.

Remark. 1. According to the Abel theorem for the exponential series in work [1] (Theorem 3.1), series (3.10) converges absolutely on a convex domain (probably, unbounded one) and uniformly on its compact subsets. This domain is determined by the Cauchy-Hadamard formula for the exponential series [1, Thm. 4.1].

2. It follows from Lemma 2.5 of work [1] that for each set of the coefficients $\left\{d_{m, l}\right\} \in Q\left(D, \Lambda_{\mathbb{Z}}\right)$ the sum $g(z)$ of series (3.10) is a function analytic in the domain $D$ (but not necessarily in the vicinity of $\bar{D})$.

\section{BIBLIOGRAPHY}

1. O.A. Krivosheyeva. A convergence domain of exponential monoms series // Ufimskij Matem. Zhurn. 3:2, 43-56 (2011). [Ufa Math. J. 3:2, 42-55 (2011).]

2. A.F. Leontiev. Exponential series. Nauka, Moscow (1976). (in Russian).

3. B.Ya. Levin. Distribution of the zeros of entire functions. Fizmatgiz, Moscow (1956). [Mathematische Lehrbücher und Monographien. II. Abt. Band 14. Akademie-Verlag, Berlin (1962). (in German).]

4. A.S. Krivosheyev, O.A. Krivosheyeva. A closedness of set of Dirichlet series sum // Ufimskij Matem. Zhurn. 5:3, 96-120 (2013). [Ufa Math. J. 5:3, 94-117 (2013).]

5. A.I. Abdulnagimov, A. S. Krivoshyev. Properly distributed subsequence on the line // Ufimskij Matem. Zhurn. 7:1, 3-12 (2015). [Ufa Math. J. 7:1, 3-12 (2015).]

6. A.S. Krivosheev, O.A. Krivosheev. Fundamental principle and a basis in invariant subspaces // Matem. Zametki. 95:5, 684-697 (2016). [Math. Notes. 99:5, 685-696 (20160.]

7. A.S. Krivosheev. A fundamental principle for invariant subspaces in convex domains // Izv. RAN. Ser. Matem. 68:2, 71-136 (2004). [Izv. Math. 68:2, 291-353 (2004).]

8. O.A. Krivosheyeva. Singular points of the sum of exponential monomials series on the boundary of convergence domain // Alg. Anal. 23:2, 162-205. [St. Petersburg Math. J. 23:2, 321-350 (2012).]

9. O.A. Krivosheeva, A.S. Krivosheev. Singular points of the sum of a Dirichlet series on the convergence line // Funkts. Anal. Pril. 49:2, 54-69 (2015). [Funct. Anal. Appl. 49:2, 122-134 (2015).]

Aidar Irekovich Abdulnagimov,

Ufa State Aviation Technical University,

Karl Marx str. 12, building 1,

450000, Ufa, Russia

E-mail: buffonishe@mail.ru

Alexandr Sergeevich Krivosheev,

Institute of Mathematics, Ufa Scientific Center, RAS,

Chernyshevsky str. 112,

450008, Ufa, Russia

E-mail: kriolesya2006@yandex.ru 